\title{
Extreme Ultraviolet Variability Experiment (EVE) Multiple EUV Grating Spectrographs (MEGS): Radiometric Calibrations and Results
}

\author{
R.A. Hock · P.C. Chamberlin • T.N. Woods • D. Crotser • \\ F.G. Eparvier · D.L. Woodraska • E.C. Woods
}

Received: 6 October 2009 / Accepted: 20 January 2010 / Published online: 24 February 2010

(c) The Author(s) 2010. This article is published with open access at Springerlink.com

\begin{abstract}
The NASA Solar Dynamics Observatory (SDO), scheduled for launch in early 2010, incorporates a suite of instruments including the Extreme Ultraviolet Variability Experiment (EVE). EVE has multiple instruments including the Multiple Extreme ultraviolet Grating Spectrographs (MEGS) A, B, and P instruments, the Solar Aspect Monitor (SAM), and the Extreme ultraviolet SpectroPhotometer (ESP). The radiometric calibration of EVE, necessary to convert the instrument counts to physical units, was performed at the National Institute of Standards and Technology (NIST) Synchrotron Ultraviolet Radiation Facility (SURF III) located in Gaithersburg, Maryland. This paper presents the results and derived accuracy of this radiometric calibration for the MEGS A, B, P, and SAM instruments, while the calibration of the ESP instrument is addressed by Didkovsky et al. (Solar Phys., 2010, doi:10.1007/s11207-009-9485-8). In addition, solar measurements that were taken on 14 April 2008, during the NASA 36.240 sounding-rocket flight, are shown for the prototype EVE instruments.
\end{abstract}

Keywords SDO $\cdot$ EVE $\cdot$ Solar EUV irradiance $\cdot$ Calibration $\cdot$ Synchrotron

\section{Introduction}

The Solar Dynamics Observatory (SDO) is the first mission in NASA's Living With a Star (LWS) program. The goal of SDO is to quantify and understand the causes of solar variability and its effects on Earth. The Extreme Ultraviolet Variability Experiment (EVE), one

The Solar Dynamics Observatory

Guest Editors: W. Dean Pesnell, Phillip C. Chamberlin, and Barbara J. Thompson.

R.A. Hock $(\bowtie) \cdot$ T.N. Woods · D. Crotser · F.G. Eparvier · D.L. Woodraska

Laboratory for Atmospheric and Space Physics, 1234 Innovation Drive, Boulder, CO 80303, USA

e-mail: rachel.hock@lasp.colorado.edu

P.C. Chamberlin

Solar Physics Laboratory, Code 671, NASA Goddard Space Flight Center, Greenbelt, MD 20771, USA

E.C. Woods

Rhodes College, 2000 North Parkway, Memphis, TN 38112, USA 
of the three experiments on SDO, will measure the variability of the Sun's irradiance in the X-ray ultraviolet (XUV) and extreme ultraviolet (EUV) wavelengths from 0.1 to $106 \mathrm{~nm}$ plus the hydrogen Lyman- $\alpha$ emission line at $121.6 \mathrm{~nm}$. The EVE irradiance measurements will be made with better than $25 \%$ accuracy over the mission lifetime.

Details about EVE can be found in Woods et al. (2010), which includes the science (measurements and modeling) goals, as well as the details of the EVE instrument design and data products. The calibration and algorithms of the Multiple EUV Grating Spectrographs (MEGS) A, B, P, and Solar Aspect Monitor (SAM) instruments are the focus of this paper. The results shown are from the final EVE calibration performed at the National Institute of Standards and Technology (NIST) Synchrotron Ultraviolet Radiation Facility III (SURF III) in Gaithersburg, Maryland, during August 2007. Also included are results from a January 2009 calibration of the prototype EVE instrument that will be flown as an underflight calibration three months after SDO launches. A brief overview of the EVE instruments is given in Section 2, followed by a discussion of EVE's calibration heritage in Section 3. The SURF III radiometric calibration facilities are detailed in Section 4, then the calibration algorithms and results of the MEGS A and B instruments are presented in Section 5, followed by a discussion of the MEGS P and SAM calibrations in Sections 6 and 7, respectively. The solar EUV spectral irradiance from a sounding-rocket flight on 14 April 2008 using the prototype EVE instrument is then presented in Section 8, followed by concluding remarks in Section 9.

\section{EVE Instruments}

The EVE instrument suite will measure the solar spectral irradiance from 0.1 to $6 \mathrm{~nm}$ with $1-\mathrm{nm}$ spectral resolution, 6 to $106 \mathrm{~nm}$ with $0.1-\mathrm{nm}$ resolution, and the hydrogen Lyman- $\alpha$ line at $121.6 \mathrm{~nm}$ with 1-nm resolution. Solar observations will be taken continuously with a ten-second cadence except during satellite eclipse periods and planned calibration activities, which are expected to total less than $1 \%$ of the mission lifetime. To cover the entire spectral range and meet accuracy requirements, EVE is composed of multiple instruments. The EVE instrument design is described in detail by Woods et al. (2010), so only a brief overview of the instruments is given here.

The primary, high-spectral-resolution irradiance measurements are made by the MEGS A and B instruments. MEGS A is an off-Rowland-circle grazing-incidence spectrograph covering the 6 to $37 \mathrm{~nm}$ range. The entrance aperture of MEGS A includes two slits: denoted A1 and A2. Light illuminating these slits shares the same grating and CCD detector, but has light paths that are offset in the cross-dispersion direction of the grating and therefore do not overlap on the CCD. In addition, MEGS A1 and A2 have separate primary filters with different spectral bandpasses to isolate different portions of the MEGS A wavelength range and reduce the effects from higher orders. MEGS A1 is optimized for the 6 to $18 \mathrm{~nm}$ range, while MEGS A2 covers 16 to $37 \mathrm{~nm}$. MEGS B is a two-grating, cross-dispersing spectrograph covering the 36 to $106 \mathrm{~nm}$ range. The two orthogonal gratings are designed to eliminate the out-of-band light without the use of a bandpass filter and to disperse the higher-order spectra off the primary first-order spectrum.

Both MEGS A and B use the same type of $2048 \times 1024$ CCD. Westhoff et al. (2007) provide more information about the CCDs used with MEGS. Ray-tracing results for the MEGS spectrometers are found in Crotser et al. (2004, 2007). The MEGS CCDs are twodimensional arrays. One direction, the dispersion direction, covers the wavelength range of the instrument over 2048 pixels. The other direction, the cross-dispersion direction, images the slit at each wavelength over 1024 pixels. As a result, the solar irradiance for any given 
wavelength is spread over many pixels in the cross-dispersion direction of the CCD. We create our final science products by adding together all pixels with a certain wavelength.

MEGS P measures the Lyman- $\alpha$ emission with 0.25 -second cadence using a Si photodiode. Lyman- $\alpha$ is isolated by placing the diode in the MEGS B optical path at the point where $121.6 \mathrm{~nm}$ photons are dispersed after MEGS B's first grating. A limiting bandpass Lyman- $\alpha$ filter is also used to eliminate potentially significant out-of-band light.

Included as part of the MEGS A package is SAM: a pinhole camera that observes the short XUV wavelengths $(0.1-7 \mathrm{~nm})$. SAM is designed to record single photon events that can be used to reconstruct a spectrum with 1-nm spectral resolution. In addition, SAM has the ability to generate images with a coarse spatial resolution of approximately 15 arcseconds per pixel.

The EUV SpectroPhotometer (ESP) instrument uses a transmission grating to disperse EUV light onto a set of broadband EUV photometers. This instrument will not only get higher temporal resolution measurements ( 0.25 seconds), but will also, along with underflight calibration rockets, help quantify and track the long-term degradation of the MEGS instruments. As ESP's diodes are expected to degrade slowly over time, they can help to re-calibrate MEGS results if MEGS undergoes a sudden change in responsivity from a CCD bakeout or science-filter failure. While the absolute calibration of EVE will be tied to the planned annual rocket underflights, ESP and MEGS P can help track degradation trends between rocket flights. The details of the ESP instrument are presented by Didkovsky et al. (2010).

All instruments on EVE have a rotatable filter wheel in front of their entrance apertures. These filter wheels house redundant science filters, which may be necessary in the event that a primary science filter develops pinholes. There are also blank filter positions that allow for dark measurements on orbit. One filter position in each of the MEGS A and B filter holders contains a "second-order filter" that blocks the short-wavelength portion of the primary filter and quantifies the effects of higher grating orders. Other positions have filters that transmit visible light to check for out-of-band scattered light. Triplett et al. (2007) give calibration results for the EVE filters.

\section{EVE MEGS Calibration Heritage}

The MEGS instruments on EVE are a follow-on to the Solar EUV Experiment (SEE) that has been making measurements since February 2002 of the solar spectral irradiance from 0.1 to $195 \mathrm{~nm}$ onboard the Thermosphere, Ionosphere, Mesosphere Energetics and Dynamics (TIMED) satellite (Woods et al., 2005). Although EVE has a limited spectral range compared to SEE, EVE will improve upon the temporal and spectral resolution, accuracy, and duty cycle. Currently, SEE is planned to overlap with EVE in time to compare the absolute values of the two experiments and create a continuous record of the solar EUV irradiance.

SEE has provided experience not only in designing and building the EUV spectrographs and photometers, but also in determining the radiometric calibration. For example, the $E U V$ Grating Spectrograph (EGS), an instrument on SEE and a copy for underflight rocket payloads, has been calibrated at SURF more than eight times. This includes two calibrations of the flight EGS before launch as well as several calibrations of the rocket underflight copy. Another instrument, the X-ray Photometer System (XPS) has been flown as different versions on the Student Nitric Oxide Experiment (SNOE; Bailey et al., 2006), TIMED (Woods et al., 2005), and the Solar Radiation and Climate Experiment (SORCE; Woods and Rottman, 2005) as well as a prototype version that has flown on various sounding rocket 
flights. As a result, it has been calibrated at SURF numerous times. The TIMED/SEE calibrations and algorithms are described by Woods et al. (2005), while the SORCE/XPS calibrations and results are given by Woods, Rottman, and Vest (2005).

\section{SURF Calibration Setup}

The primary EVE calibrations are performed in the vacuum chamber at the end of beam line 2 (BL-2) at the SURF III calibration facility in Gaithersburg, Maryland. SURF provides a primary radiometric source that is accurate to $1 \%$, one of the most accurate UV sources available (Arp et al., 2000). There are certain advantages of using this primary source over using secondary, transfer standards that were first calibrated at NIST. Calibrating at SURF not only eliminates the uncertainties associated with using a transfer standard but also provides an adjustable source in both intensity and wavelength that provides flexibility to optimize many of the calibrations discussed throughout this paper. The key calibration parameters for the SURF beam are the beam energy, which determines the peak wavelength, and the beam current, which determines the intensity.

At SURF, the EVE instruments are coherently illuminated by the synchrotron radiation from the electrons that travel around the SURF ring with a frequency of $57 \mathrm{MHz}$. The diffraction effects from BL-2 baffles are minimized to less than $1 \%$ for the EVE calibrations. First, by having the EVE entrance slit smaller than the SURF illuminated beam size and also having the slit located in the center of the beam, the beam line baffle diffraction patterns do not enter the MEGS slits. Furthermore, the EVE grating is much larger than the slit diffraction pattern incident on the grating; thereby, the majority of the radiation reaches the detector. Consequently, no corrections for diffraction are made in the MEGS algorithms.

EVE is mounted in a vacuum chamber that allows the entire system, from electron ring to instrument CCDs, to be in a vacuum environment, thus eliminating any influence or absorption of the EUV photons by air or glass windows. Inside the vacuum chamber, EVE is mounted to a gimbal system that provides pitch and yaw movements. This allows the fieldof-view (FOV), or the angle of the SURF beam relative to the normal of the entrance slit, to vary. As the SURF beam is much smaller that the angular size of the Sun, it is important to vary the FOV to characterize the resulting changes in the optical responses. The vacuum chamber also allows for $x$ - and $y$-translations in order to center the SURF beam in the middle of the aperture for each EVE instrument.

Both the beam energy and current determine the photon flux from the SURF beam. The beam energy determines the spectral shape, while the current determines the intensity. Figure 1 shows the photon flux per milliamp of SURF current for each beam energy used in the EVE calibrations. Lower energies shift the peak intensity to longer wavelengths and have fewer photons per unit current. As a result, using lower energies requires higher currents to provide a similar photon flux into the instrument. Most calibrations were done using the $380 \mathrm{MeV}$ beam energy. The current was adjusted to get high counts but not so high as to saturate any of the CCD's pixels. Correcting for the effects of higher orders requires using multiple energies; the details of this process are discussed in Section 5.4.4.

In general, to improve the counting statistics and reduce uncertainties, at least 20 tensecond images are co-added for each calibration test. Furthermore, each instrument is calibrated separately to allow us to optimize the beam current and energy. Once all of the calibration data have been taken, these data need to be processed to obtain the final responsivity for each of the EVE instruments. 
Figure 1 The SURF flux for beam energies from $140 \mathrm{MeV}$ to $380 \mathrm{MeV}$.

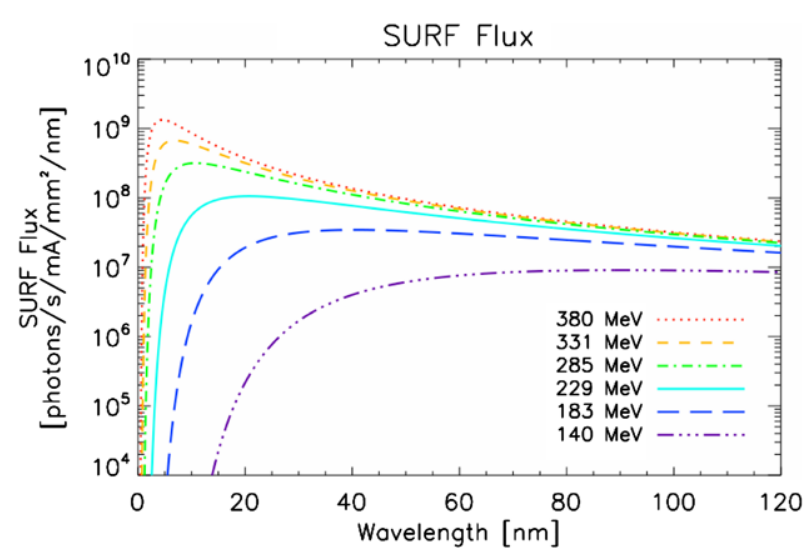

\section{MEGS A and B Calibration}

As with any spectrograph, the many instrument parameters require calibration and/or analysis in order to use the spectrograph's data for scientific results in meaningful units. In the case for MEGS A and B, the primary result is the solar spectral irradiance, which we plan to report in units of $\mathrm{W} \mathrm{m}^{-2} \mathrm{~nm}^{-1}$. Passing through the light path, the following instrument parameters are briefly introduced: Firstly, the entrance-slit area is needed to provide the $\mathrm{m}^{-2}$ part of the measured irradiance. Then the incoming light is transmitted through a filter, diffracted off a grating into individual wavelengths, and absorbed in a CCD pixel where electron-hole pairs are created.

The efficiency of each of these optical elements can be calibrated separately, but in the case of the instrument-level calibrations performed at SURF, the net effect of all of the optics is calibrated simultaneously to derive the MEGS responsivity. A critical part of this responsivity is accurately knowing the incoming irradiance, or flux, of the light source; in this case, the SURF synchrotron irradiance is known with a relative accuracy of about $1 \%$. For this analysis, the responsivity $[R]$ will be given in units of data number [DN] per photon. The detector's signal, in DN, is actually the conversion of the number of electrons detected in each pixel over the integration period. This electronic conversion, enabled by charge amplifier and analog-to-digital converter [ADC], is referred to as gain. The CCD detector's gain per pixel varies slightly with temperature and is dependent on which charge amplifier chain or readout mode is used in reading the CCD pixels. Therefore, the MEGS calibration measurements at SURF are taken at different temperatures and using all readout modes to determine the variations in gain. Another important parameter for the CCD readout is the detector background (dark) signal, which also varies with temperature and readout mode. The angle of the incoming radiation affects where the light falls on the optical elements (filter, grating, CCD), so the responsivity as a function of incoming angle requires careful calibration. The mapping of responsivity over the instrument field-of-view (FOV) is an important part of the SURF calibrations, so that the SURF measurements, which are made with a small beam of light, can be properly averaged for the larger angular size of the Sun and in order to characterize how the responsivity changes with any offset pointing of the Sun from the ideal MEGS optical center point. The final part of the MEGS calibration needed for the spectral-irradiance calculation is specifying the spectral bandpass per CCD pixel, which is accomplished with wavelength-scale calibrations and raytrace analysis of the spectrograph design. 
While MEGS A and B have different optical designs as discussed in Section 2 and in more detail by Woods et al. (2010), the data from these instruments are calibrated and processed in the same way, as both instruments have the same type of CCDs. This section presents the algorithms and calibration results for MEGS A1, A2, and B. The first subsection, Section 5.1, discusses the algorithms necessary to convert CCD detector counts in DN to irradiance in $\mathrm{W} \mathrm{m}^{-2} \mathrm{~nm}^{-1}$. Section 5.2 discusses the determination of the wavelength scale and spectral resolution, while Sections 5.3 and 5.4 present the details of the algorithms and calibration results. While the focus of this paper is on the calibration of the EVE instruments to be flown on SDO, final calibration results for the prototype EVE instrument flown on a NASA sounding rocket in 2008 are included for comparison. Some early calibration results for MEGS are presented by Chamberlin et al. (2007, 2009).

\subsection{MEGS A and B Algorithms}

There are two aspects of the MEGS algorithms: One is the responsivity algorithm using the SURF calibration measurements, and the other is the irradiance algorithm for the solar observations. These two algorithms are in concept identical, but inverted, equations. For the SURF calibrations, the responsivity is calculated as the measured signal divided by the known synchrotron irradiance. For the solar observations, the solar spectral irradiance is calculated as the measured signal divided by the instrument responsivity derived from SURF calibrations. In actuality, the algorithms are more detailed as the responsivity parameters are dependent on temperature and field-of-view angle, as well as other effects such as grating higher-order contributions to the measured signals. The following describes the algorithms for the MEGS signal, responsivity, and solar irradiance. The many parameters in these algorithms require characterization, from direct calibration and/or from analysis. The subsequent sections describe the calibration techniques and results for each of the primary instrument parameters.

For MEGS A and B, the responsivity is calculated for each individual pixel on the $2048 \times 1024$ CCD detectors, denoted by the indices $i$ and $j$ in the following equations. This pixel-based calibration allows us to bypass the flatfield correction sometimes used for array detectors. Furthermore, the calibration is not based on calibrations of each individual optical element of the instrument, but it is for the complete end-to-end optical system, including filter transmissions as well as grating and detector efficiencies.

The algorithms for the rocket instrument (prototype EVE) are essentially the same as those for the flight EVE. As the rocket version of MEGS does not have a filter wheel, calibrations were done for the primary (and only) filter, which is the same type as the primary science filters on the EVE flight instrument.

The first step for processing all MEGS A and B images, taken either at SURF or on orbit for solar observations, is to correct the raw images to get the corrected count rate $\left[C^{\prime}\right]$ in DN second ${ }^{-1}$ :

$$
\begin{aligned}
C^{\prime}(i, j, t)= & {\left[\frac{C\left(i, j, t, T_{\mathrm{CCD}}, \text { tap }\right)}{\Delta t}-D\left(i, j, T_{\mathrm{CCD}}, C, \text { tap }\right)\right] } \\
& \times G\left(i, j, T_{\mathrm{CCD}}, \operatorname{tap}\right) \operatorname{Mask}(i, j, C, \text { tap })
\end{aligned}
$$

The raw counts $[C]$ in each pixel are corrected for the integration time $[\Delta t]$, dark offset $[D]$, and the dependence of the CCDs on the temperature and readout mode [tap] of the electronics $[G]$. In addition, invalid pixels are identified and set to zero by applying a binary mask [Mask], allowing them to be ignored in later steps. While there are very few defective pixels 
on the CCDs, there are several pixels contaminated in each image by cosmic-ray particle hits during ground calibration and also by energetic particle hits during flight. The details of how each of these corrections are calculated will be discussed in Section 5.3.

Images taken at SURF are further processed to determine the SURF response $\left[R_{\mathrm{SURF}}\right]$ in DN photon $^{-1}$ :

$$
R_{\mathrm{SURF}}\left(i, j, E_{\text {beam }}, \text { filter, } \alpha, \beta\right)=\frac{\frac{1}{n} \sum_{k=1}^{n} \frac{C_{k}^{\prime}\left(i, j, t, E_{\text {beam }}, \text { filter }, \alpha, \beta\right)}{I_{\mathrm{SURF}}(t)}}{F_{\mathrm{SURF}}\left(i, j, E_{\text {beam }}, \alpha, \beta\right) A_{\text {slit }} \Delta \lambda(i, j)}
$$

Each corrected image $\left[C^{\prime}\right]$ is normalized by the SURF beam current $\left[I_{\mathrm{SURF}}\right]$ in $\mathrm{mA}$. Then, all images taken with the same beam energy $\left[E_{\text {beam }}\right]$, filter, and field-of-view pointing $[\alpha, \beta]$ are co-added to reduce the uncertainty. Finally, this co-added image is divided by the SURF beam flux $\left[F_{\text {SURF }}\right]$ in photons $\mathrm{s}^{-1} \mathrm{~mA}^{-1} \mathrm{~mm}^{-2} \mathrm{~nm}^{-1}$, area of the limiting aperture $\left[A_{\text {slit }}\right]$ in $\mathrm{mm}^{2}$, and bandpass $[\Delta \lambda]$ in $\mathrm{nm}$ to derive the SURF response $\left[R_{\mathrm{SURF}}\right]$.

The corrected counts used to determine the SURF response in Equation (2) can contain both first order and higher orders. As shown later, MEGS B SURF calibrations have essentially no higher-order contributions, so Equation (2) can be directly used for MEGS B. MEGS A responsivities do have contributions from higher orders so $R_{\mathrm{SURF}}$ needs to be adjusted to include only the first-order response. Equation (5) shows the relationship between $R_{\mathrm{SURF}}$ and true first-order response $\left[R_{1}\right]$ as derived from:

$$
R_{\mathrm{SURF}}\left(i, j, E_{\text {beam }}, \text { filter, } \alpha, \beta\right)=\sum_{k=1}^{m} \frac{1}{k} \frac{F_{\mathrm{SURF}}^{k}\left(i, j, E_{\text {beam }}, \alpha, \beta\right)}{F_{\mathrm{SURF}}^{k=1}\left(i, j, E_{\text {beam }}, \alpha, \beta\right)} R_{k}(i, j, \text { filter, } \alpha, \beta)
$$

where $k$ denotes the order and

$$
F_{\mathrm{SURF}}^{k}(\lambda)=F_{\mathrm{SURF}}\left(\frac{\lambda}{k}\right)
$$

The contributions of the higher orders are found and separated by a proven method that utilizes multiple beam energies at SURF (Saloman, 1975; Rottman, Woods, and Sparn, 1993; Chamberlin, Woods, and Eparvier, 2002). This multiple-beam-energy method exploits the differences in the spectral distribution of SURF fluxes for different beam energies to vary $R_{\text {SURF. }}$. Using Equation (3), a system of linear equations can be written and then solved numerically to determine each $R_{k}$. By using two different SURF beam energies, first and second order can be separated; using three energies, it is possible to calculate the contributions from first, second, and third orders. The results of this order-sorting correction are applied as a scalar correction to $R_{\mathrm{SURF}}$ :

$$
f_{\mathrm{OS}}\left(i, j, E_{\text {beam }}, \text { filter, } \alpha, \beta\right)=\frac{R_{1}(i, j, \text { filter, } \alpha, \beta)}{R_{\mathrm{SURF}}\left(i, j, E_{\text {beam }}, \text { filter, } \alpha, \beta\right)}
$$

Determining $R_{\text {SURF }}$ and the higher-order correction will be discussed in Section 5.4.

For processing solar data, the SURF response is used to calculate the flight responsivity $\left[R_{\text {flight }}\right]$ in $\mathrm{DN} \mathrm{s}^{-1}\left(\mathrm{~W} \mathrm{~m}^{-2} \mathrm{~nm}^{-1}\right)^{-1}$ :

$$
\begin{aligned}
& R_{\text {flight }}(i, j, \text { filter }) \\
& =\frac{\lambda(i, j)}{h c}\left[\sum_{\alpha, \beta} w(\alpha, \beta) R_{\mathrm{SURF}}\left(i, j, E_{\text {beam }}, \text { filter, } \alpha, \beta\right) f_{\mathrm{OS}}\left(i, j, E_{\text {beam }}, \text { filter, } \alpha, \beta\right)\right] \\
& \quad \times A_{\text {slit }} \Delta \lambda(i, j)
\end{aligned}
$$


Table 1 Example FOV map weights for solar observations. These weights are appropriate for solar observations when the on-orbit pointing is at the optical center.

\begin{tabular}{rrlll}
\hline & & $\alpha\left[^{\circ}\right]$ & & \\
\cline { 3 - 5 } & & -0.5 & 0.0 & +0.5 \\
\hline$\beta\left[^{\circ}\right]$ & +0.5 & 0.0249 & 0.1455 & 0.0249 \\
& 0.0 & 0.1455 & 0.3180 & 0.1455 \\
& -0.5 & 0.0249 & 0.1455 & 0.0249 \\
\hline
\end{tabular}

where $h c / \lambda$ converts from photon units to energy units, and $R_{\mathrm{SURF}}, f_{\mathrm{OS}}, A_{\text {slit }}$, and $\Delta \lambda$ are the SURF response, order-sorting correction, slit area, and bandpass as described above. The weight of each SURF FOV point, given by $w$, is used to average the SURF FOV map over the approximately $0.5^{\circ}$ FOV of the Sun. As mentioned in Section 4, the SURF beam is significantly smaller in angular diameter than the full-disk Sun. By correctly weighting SURF responses taken at different angles, it is possible to combine the SURF responses to determine the appropriate weighted response for the solar measurements. Table 1 gives the values of $w$ for the expected on-orbit pointing. The FOV maps are discussed in Section 5.4.3.

Next, the corrected count rate $\left[C^{\prime}\right.$ : Equation (1)] for solar data is converted to irradiance:

$$
I(i, j, t)=\frac{C^{\prime}(i, j, t, \text { filter })}{R_{\text {flight }}(i, j, \text { filter })} f_{\text {degrad }}(i, j, t, \text { filter }) f_{1 \mathrm{AU}}(t)
$$

where $R_{\text {flight }}$ is the flight responsivity defined in Equation (6), $f_{\text {degrad }}$ is the degradation correction, and $f_{1 \mathrm{AU}}$ is the correction to normalize the irradiance to $1 \mathrm{AU}$ to account for the eccentricity of Earth's orbit. The final step is to convert the irradiance image (as all calibrations are applied on the pixel level) to get a spectrum of irradiance versus wavelength. This is done by adding together all pixels whose wavelength falls within a $0.02 \mathrm{~nm}$ wavelength bin of the final spectrum; note that there are multiple wavelength bins within the spectral resolution of $0.1 \mathrm{~nm}$.

We must carefully track and propagate the uncertainties in all of the algorithms. The details of the uncertainties for each correction will also be discussed in the appropriate sections. Here, we simply present the equations used to propagate the uncertainties that determine the irradiance accuracy. The uncertainty in $C^{\prime}$ [Equation (1)] is given by:

$$
\sigma_{C^{\prime}}^{2}=\left(C^{\prime}\right)^{2}\left[\frac{\left(\frac{C}{\Delta t}\right)^{2}\left(\frac{\sigma_{C}^{2}}{(C)^{2}}+\frac{\sigma_{\Delta t}^{2}}{(\Delta t)^{2}}\right)+\sigma_{D}^{2}}{\left(\frac{C}{\Delta t}-D\right)^{2}}+\frac{\sigma_{G}^{2}}{(G)^{2}}\right]
$$

while the uncertainty in $R_{\mathrm{SURF}}$ [Equation (2)] is given by

$$
\sigma_{R_{\mathrm{SURF}}}^{2}=\left(R_{\mathrm{SURF}}\right)^{2}\left[\frac{\frac{1}{n^{2}} \sum_{k=1}^{n}\left(\frac{C_{k}^{\prime}}{I_{\mathrm{SURF}}}\right)^{2}\left[\frac{\sigma_{C^{\prime}}^{2}}{\left(C_{k}^{\prime}\right)^{2}}+\frac{\sigma_{I_{\mathrm{SURF}}}^{2}}{\left(I_{\mathrm{SURF}}\right)^{2}}\right]}{\left(\frac{1}{n} \sum_{k=1}^{n} \frac{C^{\prime}}{I_{\mathrm{SURF}}}\right)^{2}}+\frac{\sigma_{F_{\mathrm{SURF}}}^{2}}{\left(F_{\mathrm{SURF}}\right)^{2}}\right]
$$

Slit area $\left[A_{\text {slit }}\right]$ and bandpass [ $\left.\Delta \lambda\right]$ do not contribute to the uncertainty of $R_{\text {flight }}$ as the terms cancel out in Equations (2) and (6). The uncertainty for the order-sorting correction (Equation (5)) is found by comparing order-sorting results using different combinations of beam energies. This is discussed in more detail in Section 5.4.4. Then, the uncertainty in $R_{\text {flight }}$ [Equation (6)] is given by:

$$
\sigma_{R_{\text {flight }}}^{2}=\left(R_{\text {flight }}\right)^{2}\left[\frac{\sigma_{\lambda}^{2}}{(\lambda)^{2}}+\frac{\sum_{\alpha, \beta}\left(w R_{\mathrm{SURF}} f_{\mathrm{OS}}\right)^{2}\left[\frac{\sigma_{w}^{2}}{(w)^{2}}+\frac{\sigma_{R_{\mathrm{SURF}}}^{2}}{\left(R_{\mathrm{SURF}}\right)^{2}}+\frac{\sigma_{f_{\mathrm{OS}}}^{2}}{\left(f_{\mathrm{OS}}\right)^{2}}\right]}{\left(\sum_{\alpha, \beta} w R_{\mathrm{all}} f_{\mathrm{OS}}\right)^{2}}\right]
$$


Finally, the irradiance accuracy using Equation (7) is given by:

$$
\sigma_{I}^{2}=I^{2}\left[\frac{\sigma_{C^{\prime}}^{2}}{\left(C^{\prime}\right)^{2}}+\frac{\sigma_{R_{\text {flight }}}^{2}}{\left(R_{\text {flight }}\right)^{2}}+\frac{\sigma_{f_{\text {degrad }}}^{2}}{\left(f_{\text {degrad }}\right)^{2}}+\frac{\sigma_{f_{1 \mathrm{AU}}}^{2}}{\left(f_{1 \mathrm{AU}}\right)^{2}}\right]
$$

The following subsections describe the calibration technique and results for determining each of these parameters in the MEGS algorithms.

\subsection{Wavelength Scale, Bandpass, and Spectral Resolution}

Before any radiometric calibrations are performed, the wavelength scale for each pixel on the CCDs must be determined. The wavelength scale or the wavelength associated with each pixel on the CCD is used to determine the SURF flux illuminating each pixel. It is also used when converting an irradiance image into a spectrum.

Because the primary calibration facility, SURF, is a continuum source, measuring a definitive wavelength scale is not straightforward. Instead, a more accurate method was employed prior to going to SURF by illuminating MEGS A and B with well-known emission spectra from various gases using a hollow-cathode lamp.

\subsubsection{LASP Calibration Setup}

The Multiple Optical Beam Instrument (MOBI) vacuum chamber at the Laboratory for Atmospheric and Space Physics (LASP) in Boulder, Colorado incorporates a hollow-cathode lamp capable of generating emission-line spectra from the visible down to $\approx 25 \mathrm{~nm}$ using various gases including neon, argon, helium, and nitrogen. These spectral lines provide focus and alignment information, as well as provide a method to determine the wavelength scale and spectral resolution of MEGS A and B.

The hollow-cathode lamp is mounted to the tank with a large, flexible vacuum bellows and is differentially pumped to provide an unimpeded path for the EUV light, which would otherwise be absorbed by air or glass windows. One side of the bellows is mounted to an external $x-y$ translation system that allows $\pm 4^{\circ}$ off-axis illumination in both directions for focus and alignment activities. EVE is mounted to a vacuum $x$-stage inside MOBI, which allows for additional off-axis maneuvers as well as illumination of all EVE instruments without breaking vacuum and repositioning the experiment.

\subsubsection{Wavelength Scale}

With EVE in the MOBI vacuum chamber, emission spectra are observed for each of the instruments. For each spectrum, a subset of bright lines is selected whose wavelengths are known. The profile of each line is fit to a Gaussian to determine the pixel location of the peak. Then a high-order polynomial is used to fit wavelength versus pixel location in the dispersion direction. Each row (cross-dispersion direction) is fit independently to allow for any curvature of the slit image on the detector. This process is repeated for different pointing angles to determine how the wavelength scale varies with field-of-view. These fits of the wavelength scale are verified by ray tracing the optical system (e.g. see Crotser et al. 2004, 2007).

Once on orbit, the solar-irradiance spectrum will be used to verify the pre-flight wavelength scale. This is particularly important for MEGS A, as the shortest wavelength seen in the MOBI spectra is around $25 \mathrm{~nm}$. Therefore the extrapolation of the wavelength fit to the MEGS A spectral range down to $6 \mathrm{~nm}$ can have increased uncertainties. 

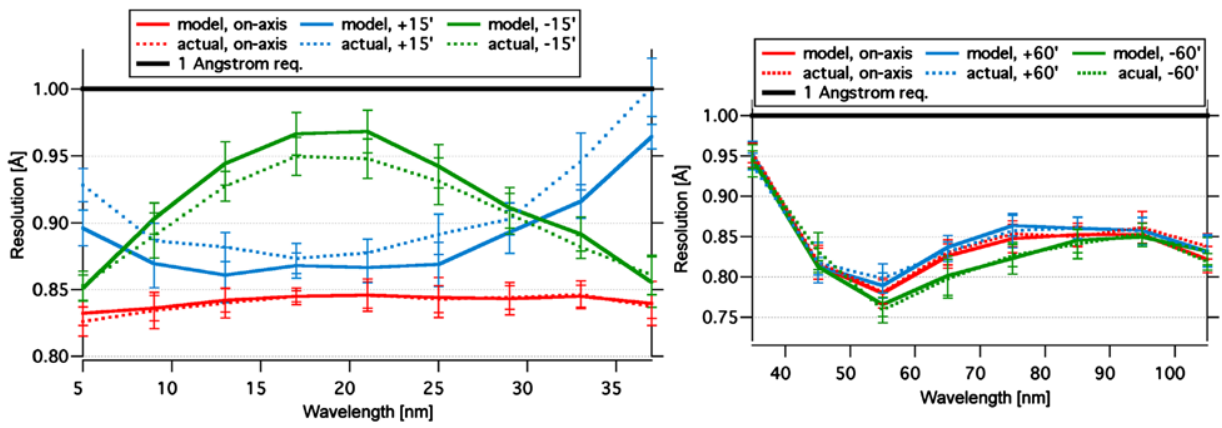

Figure 2 The expected (modeled) and measured spectral-resolution performance for MEGS A (left) and MEGS B (right). There results are for both on-axis and off-axis illumination. The measured results are based on instrument performance using the spectra produced by various hollow-cathode gases. These results indicate that the spectral resolution is better than 0.1 .

\subsubsection{Bandpass}

The bandpass $[\Delta \lambda]$ in Equations (2) and (6) is the spectral width of each pixel. The bandpass is not related to spectral resolution, which is the width of the spectral lines, but is instead related to the step size along the wavelength scale. The bandpass for pixel $(i, j)$ is defined as the absolute value of the difference between wavelength of the pixel on the left $(i-1, j)$ and the pixel on the right $(i+1, j)$ divided by two:

$$
\Delta \lambda(i, j)=\frac{|\lambda(i+1, j)-\lambda(i-1, j)|}{2}
$$

The absolute value is necessary as the wavelength of pixel does not necessary increase as the pixel indices increase.

\subsubsection{Spectral Resolution}

The spectral resolution as a function of wavelength and off-axis pointing can be determined using the MOBI spectra. The measured spectral resolution is the full width at half maximum $[\mathrm{FWHM}]$ of the Gaussian fits of the line profiles in the dispersion direction. Figure 2 shows the measured spectral resolution for MEGS as a function of wavelength as well as the modeled solar spectral resolution. These results, shown for on-axis and off-axis illumination, indicate that the measured spectral performance is comparable to the modeled performance. Furthermore, the results indicate that the spectral resolution requirement of $0.1 \mathrm{~nm}$ has been exceeded.

\subsection{Corrected Count Rate}

The first step in processing the raw image is to convert the images to raw count rates by dividing the raw counts by the integration time. The uncertainty of the raw counts, used in Equation (8), is about two data number [DN] per pixel, or four electrons per pixel. The standard integration time for MEGS images is ten seconds with an uncertainty of 0.001 second. The following sections describe generating the corrections to this count rate for MEGS A and $\mathrm{B}$ as shown in Equation (1). 
Figure 3 The thermal dark as a function of CCD temperature for one pixel on the MEGS A CCD. The shaded region shows the uncertainty in the thermal dark correction. The vertical dotted line indicates the flight operating temperature for the CCDs.

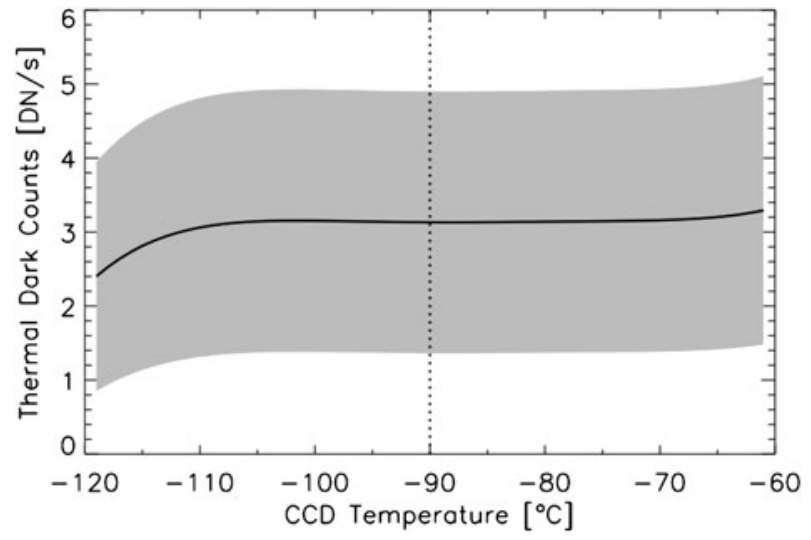

\subsubsection{Dark Count Correction}

There are two dark-rate components that need to be considered: one due to the bias in the electronics, and one due to the thermal noise of the CCDs. The electronic bias is determined by averaging the four "virtual" columns, being the first four columns in the image that read the charge amplifier signal (bias) prior to reading actual CCD pixels. In data processing, the electronic dark component is the average of the four virtual columns for each half of the CCD. Each half of the CCD is read out through different charge amplifier chains, so each CCD half has unique electronic-bias characteristics. The uncertainty of the electronic dark is the standard deviation of these pixels.

The second dark component is the thermal dark. This dark is due to the thermal noise of the CCDs and cannot be directly measured for each solar image. Instead, a fit to the thermal dark is determined by using dark images taken over a range of temperatures and repeated during several cycles as part of environmental thermal vacuum tests. The temperature behavior of each pixel is well characterized by a polynomial fit after subtracting the electronic dark in each image. Figure 3 shows the thermal dark component for one pixel in MEGS A as a function of temperature. The uncertainty in the thermal dark is determined by the goodness of the polynomial fit, and an example is given by the shaded region in Figure 3. Each CCD pixel has its own thermal dark function. The thermal dark and uncertainty are very small (1 DN is about two electrons) for these cold operating temperatures.

\subsubsection{Scattered Light}

Light scattering, primarily from the diffraction gratings but also possible from reflections off other surfaces (e.g. baffle edges, optics masks, limiting apertures), can be a significant uncertainty in spectrometers. With EVE, multiple proven techniques were used early in the design to reduce the amount of scattered light, such as using a holographically ruled grating, machining knife edges on all apertures and baffles, and inserting bandpass filters to reject out-of-band light from entering the optical cavity. As a result, there is very little measured scattered light, amounting to less that $1 \%$ of the total counts. The scattered light is therefore insignificant, and no correction for it is applied in data processing.

\subsubsection{Linearity}

An important feature of the MEGS CCDs is the high degree of linearity of their response, as expected for silicon devices. For a linear detector, the dark-corrected counts are a linear 
Figure 4 Thermal gain correction for each half of the CCDs as a function of CCD temperature. The gain correction is forced to be unity at $-85^{\circ} \mathrm{C}$. The vertical line at $-90^{\circ} \mathrm{C}$ shows where we expect to operate on orbit. As an example, the data points used for the bottom half of MEGS B are shown as black crosses.

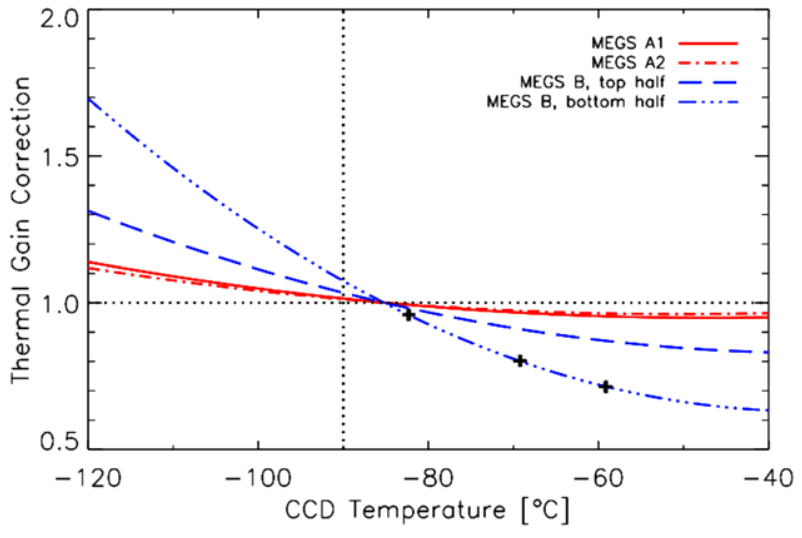

function of the input signal. The linearity of the MEGS CCDs is determined at SURF by measuring the count rate versus SURF beam current for a range of beam currents. By varying the SURF beam current by two orders of magnitude, we measure the linearity over the full dynamic range of the CCDs, which is limited by its 14-bit ADC. The residuals of a linear fit to the corrected count rate and beam current are much less than $1 \%$ for the full dynamic range of the CCDs. As the residuals are so small, there is no need to apply a linearity correction.

\subsubsection{Gain Correction}

There are two gain components that account for changes in the CCD responsivity due to temperature and readout mode of the electronics. Both of these gain components are relative, i.e. we normalize the MEGS images to a default state (CCD temperature at $-85^{\circ} \mathrm{C}$ and default readout mode). The default temperature is chosen to be $-85^{\circ} \mathrm{C}$ to balance the warmer temperatures at SURF and the colder temperatures expected on orbit. While these gain parameters are unity at the default state, the true gain of the electronics is about two electrons per DN.

The first gain component is the temperature gain. This is determined by taking data at SURF at multiple temperatures. The images are dark-corrected and normalized by the SURF beam current. These corrected counts are then divided by the corrected counts at $-85^{\circ} \mathrm{C}$ to get the normalized counts for each temperature. Then a polynomial is fit to the inverse of the normalized counts to get the relative change as a function of CCD temperature for each half of the CCD. This polynomial is the multiplicative temperature-gain correction as seen in Figure 4. The coefficients of the polynomial fits for both the flight and rocket instruments are given in Table 2. The uncertainty in the thermal gain is $1 \%$, which is the average residual of the fit.

The second gain component is associated with the readout mode of the electronics and is necessary as there are redundant charge amplifiers that have different gain factors. The default readout mode for the top CCD half is the left charge amplifier and the right amplifier is redundant. For the bottom CCD half, the default readout is the right charge amplifier and the left is redundant. The readout mode gain is calculated by averaging the ratio of coadded corrected images taken with the default readout mode to co-added corrected images taken with the redundant readout mode. For the default readout mode, this gain is unity. For MEGS A, the difference in the gain from unity for other readout modes is up to $7 \%$; for 
Table 2 MEGS CCD temperature gain coefficients. The temperature-gain function is a polynomial of the form $G=a+b(T+85)+c(T+85)^{2}$. Instances where no data are available to fit the gain are marked with an asterisk $(*)$.

\begin{tabular}{lllll}
\hline Instrument & Readout mode & $a$ & $b$ & $C$ \\
\hline Flight MEGS A & Left (top half) & 1.028 & $3.363 \times 10^{-3}$ & $3.572 \times 10^{-5}$ \\
& Right (top half) & 1.046 & $3.801 \times 10^{-3}$ & $3.832 \times 10^{-5}$ \\
& Left (bottom half) & 1.068 & $3.869 \times 10^{-3}$ & $3.612 \times 10^{-5}$ \\
& Right (bottom half) & 1.044 & $3.285 \times 10^{-3}$ & $3.251 \times 10^{-5}$ \\
Flight MEGS B & Left (top half) & 0.904 & $4.422 \times 10^{-3}$ & $6.526 \times 10^{-5}$ \\
& Right (top half) & 0.842 & $2.674 \times 10^{-3}$ & $5.327 \times 10^{-5}$ \\
& Left (bottom half) & 0.774 & $9.350 \times 10^{-3}$ & $1.409 \times 10^{-4}$ \\
& Right (bottom half) & 0.814 & $1.046 \times 10^{-3}$ & $1.484 \times 10^{-4}$ \\
Rocket MEGS A & Left (top half) & 1.007 & $8.288 \times 10^{-4}$ & $8.826 \times 10^{-6}$ \\
& Right (top half)* & 1 & 0 & 0 \\
& Left (bottom half)* & 1 & 0 & 0 \\
& Right (bottom half) & 1.003 & $6.739 \times 10^{-4}$ & $7.550 \times 10^{-6}$ \\
Rocket MEGS B & Left (top half) & 0.977 & $1.171 \times 10^{-3}$ & $1.705 \times 10^{-5}$ \\
& Right (top half)* & 1 & 0 & 0 \\
& Left (bottom half)* & 1 & 0 & 0 \\
& Right (bottom half) & 0.940 & $3.474 \times 10^{-4}$ & $1.235 \times 10^{-5}$ \\
\hline
\end{tabular}

MEGS B, it is a correction of up to $12 \%$. The uncertainty for the default readout mode is zero. For other readout modes, it is 5\%: the standard deviation of the gain ratio.

\subsubsection{Invalid Pixel Masking}

The invalid pixel mask [Mask in Equation (1)] is a combination of several binary masks that returns one if the pixel contains valid data and zero if the data are invalid. As the mask process simply identifies unusable pixels there is no uncertainty associated with it. The number of pixels eliminated by this mask is small $(<1 \%)$, although it is expected to increase for large solar storms.

The first mask is to eliminate particle hits. MEGS A and B are sensitive to cosmic-ray hits, which can be seen on the CCDs as bright dots or streaks. Particle hits are not expected to persist from one image to the next so by comparing an image to the previous image, it is possible to identify pixels with counts that are much brighter. These are identified as particle hits and are masked out.

The next type of invalid pixels is saturated pixels. It is possible that during extremely large flares some pixels on MEGS A and B may become saturated. Including these pixels would underestimate the final solar irradiance so they are masked out and excluded from processing. The columns of virtual pixels used to determine the electronic dark as discussed in Section 5.3.1 are also masked off as they do not contain valid data.

Finally, pixels that behave differently from the other pixels are masked out. These pixels are identified using the flatfield images. Both MEGS A and B have flatfield lamps that uniformly illuminate the detector. The flatfield lamps, blue and violet LEDs, were used daily during SURF calibrations and will also be used for daily in-flight calibrations. While flatfield images are usually used to correct for the pixel-to-pixel variations in order to combine 
Table 3 Uncertainty for SURF beam current. This relative uncertainty is dependent on the SURF beam configuration, such as the use of $380 \mathrm{MeV}$ and $183 \mathrm{MeV}$ beam energies, for each MEGS instrument. This uncertainty is calculated using Equation (13).

\begin{tabular}{llc}
\hline Instrument & $380 \mathrm{MeV}$ & $183 \mathrm{MeV}$ \\
\hline MEGS A1 & $0.001 \%$ & $0.4 \%$ \\
MEGS A2 & $0.003 \%$ & $0.2 \%$ \\
MEGS B & $0.1 \%$ & $0.4 \%$ \\
\hline
\end{tabular}

the pixels of similar wavelengths (Chamberlin, Woods, and Eparvier, 2002), this is not necessary for MEGS A and B as the responsivity is found at SURF for each individual pixel. However, by comparing images taken with different flatfield-lamp intensity levels, we can identify pixels that do not behave the same way as the majority and define a flatfield mask to exclude these defective pixels.

\subsection{SURF Responsivity}

The next step in processing the SURF data is to take the corrected count rate and determine the SURF responsivity. The following sections detail this calculation as shown in Equation (2). Also included are the results of the FOV maps and higher-order corrections shown in Equations (3-6).

It is important to note that after normalizing the corrected count rate by the SURF beam current, multiple images are co-added to reduce the uncertainty. In general, four minutes of ten-second integrations are co-added for each point in the FOV map. This reduces the uncertainty by a factor of almost five. For the higher-order corrections, ten minutes of data are used to reduce the uncertainty by a factor of almost eight and are necessary as some of the higher energies produce very low count rates due to the current limitations of SURF.

\subsubsection{SURF Beam Current}

For the August 2007 SURF calibrations, beam currents were recorded every five seconds. For processing the SURF data, these measurements are linearly interpolated to the middle of the ten-second CCD integration.

The uncertainty in the SURF beam current is proportional to the rate of change of the current and to the uncertainty in the timing between the SURF control computer and EVE microprocessor:

$$
\sigma_{I_{\mathrm{SURF}}}=\sigma_{t} \frac{\mathrm{d} I_{\mathrm{SURF}}}{\mathrm{d} t}
$$

Higher beam currents, which are needed with the lower SURF beam energies, decay faster so there is more uncertainty in those current measurements. Furthermore, the beam current is recorded on a separate computer from EVE. While the EVE microprocessor and the SURF control computer are synchronized to a reference time, the relative time between the computers can and does vary. The uncertainty in the timing of the SURF beam current $\left[\sigma_{t}\right]$ is estimated to be less than one second. Table 3 shows the uncertainties for both $380 \mathrm{MeV}$ and $183 \mathrm{MeV}$ beam energies for MEGS A1, A2, and B. 
Figure 5 The primary (red diamonds) and secondary (blue crosses) $3 \times 3$ FOV maps. The dispersion direction is the $\alpha$ axis, while the cross-dispersion direction is the $\beta$ axis. Both maps share a common center point.

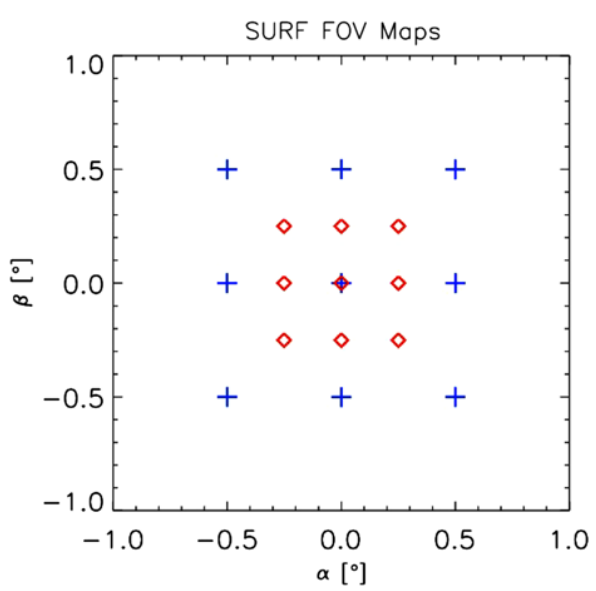

\subsubsection{SURF Flux}

The photon flux provided by SURF is provided as flux versus wavelength as seen in Figure 1. As the calibrations are calculated on a pixel basis, the provided SURF flux is interpolated onto the wavelength scale. Aided by the ability of EVE to view the primary radiometric source of SURF, the relative uncertainty in the SURF flux is 1\% (Arp et al., 2000).

\subsubsection{Field-of-View (FOV) Maps}

An important characterization is determining the responsivity of the instrument as a function of FOV as the SURF beam is significantly narrower than the Sun. The SURF beam FOV is a few arcminutes wide in the horizontal plane but is less than one arcminute vertically. The SURF responsivity is calculated for each angle in the primary and secondary $3 \times 3$ FOV maps. The primary map is a $0.5^{\circ} \times 0.5^{\circ} \mathrm{FOV}$ range with $0.25^{\circ}$ steps in each direction, with $\alpha$ defined as the dispersion direction and $\beta$ the cross-dispersion direction. The secondary map is a $1.0^{\circ} \times 1.0^{\circ} \mathrm{FOV}$ range with $0.5^{\circ}$ steps in each direction. The primary map allows for averaging over the solar FOV of about 30-arcminutes diameter and the secondary map allows for offsets from the ideal optical center. In Figure 5, the primary map angles are shown as the red diamonds, while the secondary map points are the blue crosses. Both maps share a common center so that different maps can be compared. While the primary map is sufficient if the pointing of EVE once on orbit is exactly centered, the secondary map allows EVE to make valid science measurements even if the pointing is off-center.

Figures 6, 7, and 8 show the SURF responsivity for the primary FOV map for MEGS A1, A2, and B. For each figure, the top plot shows the responsivity for each of the nine FOV angles. The red curve is the center. The bottom plot shows the relative difference between each FOV angle and the center. Overall, the differences due to FOV are less than $20 \%$. As mentioned in Section 5.2.2, the wavelength scale for MEGS A1 and A2 has higher uncertainty than for MEGS B; therefore, there may be wavelength shifts that have not been accounted for in this instrument that would contribute to these larger than expected uncertainties. MEGS B, where the wavelength scale is well understood, has even smaller FOV differences. Once on orbit, in-flight FOV maps will be obtained to validate these pre-flight maps. 
Figure 6 Primary FOV map results for MEGS A1. The top panel shows the responsivity for each of the nine FOV points. The red curve is the center point. The bottom panel shows the relative difference between each FOV point and the center point.
Figure 7 Primary FOV map results for MEGS A2. The top panel shows the responsivity for each of the nine FOV points. The red curve is the center point. The bottom panel shows the relative difference between each FOV point and the center point.
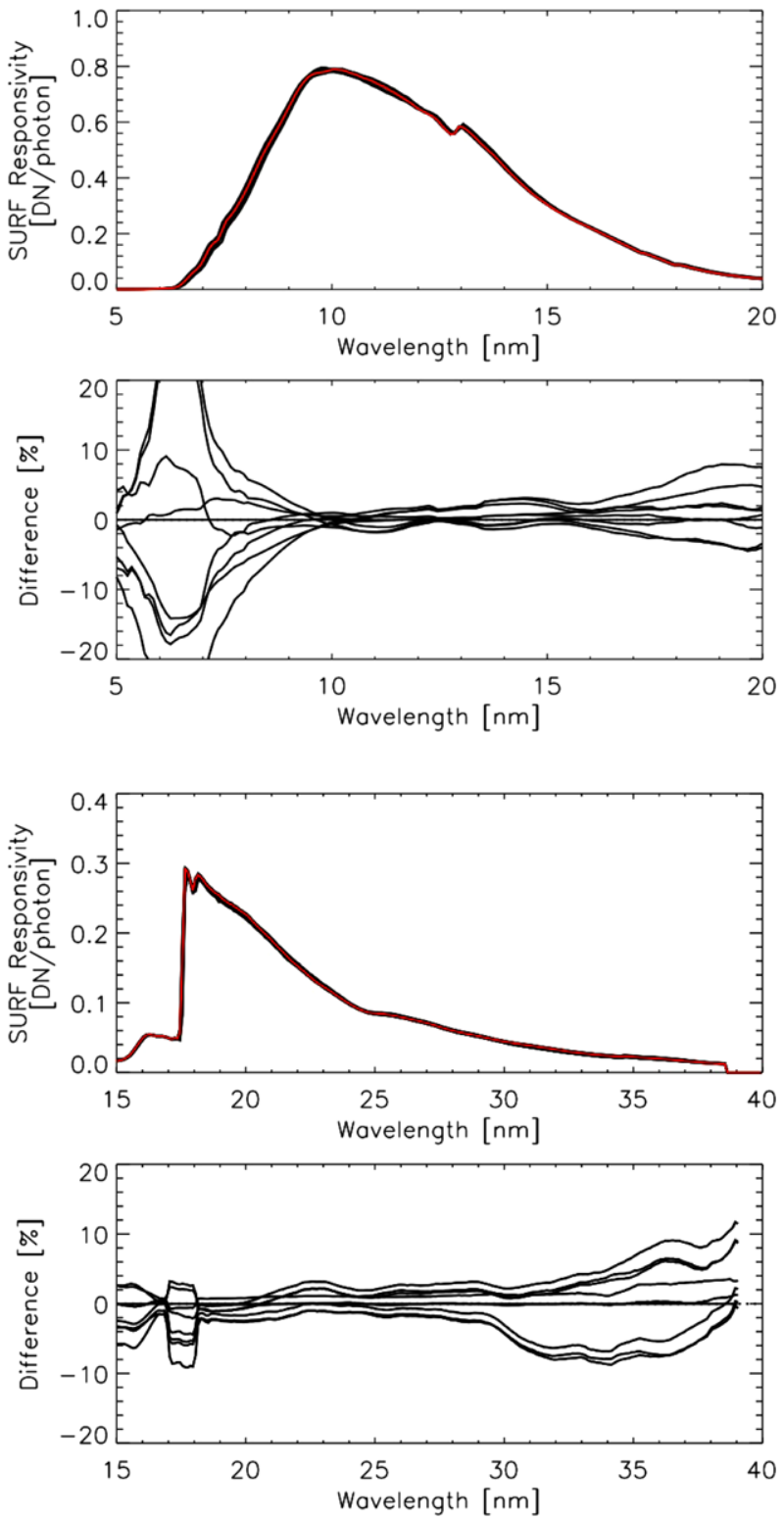

\subsubsection{Higher-Order Correction}

Correcting for contributions from higher orders is critical for meeting our uncertainty requirements. As discussed in Section 5.1, higher orders for SURF calibrations are separated by using multiple beam energies. Figures 9 and 10 show the order-sorting results for MEGS A1 and A2. We did not find any higher-order contributions for MEGS B. These results from MEGS A1, A2, and B all agree with what we expect if we calculate the higherorder contributions using the theoretical grating efficiencies, filter transmissions (A1 and A2 only), and CCD responsivity. Given these results, the optical design was successful in 
Figure 8 Primary FOV map results for MEGS B. The top panel shows the responsivity for each of the nine FOV points. The red curve is the center point. The bottom panel shows the relative difference between each FOV point and the center point.
Figure 9 Results of the multiple-beam-energy method for MEGS A1. The top panel shows the SURF responsivity without any correction (black), the true first-order responsivity (red) and the second-order responsivity (blue). The bottom panel shows the expected contribution to the first-order spectrum from the second-order spectrum at half the wavelength. MEGS A1 is intended for solar measurements below $17 \mathrm{~nm}$.
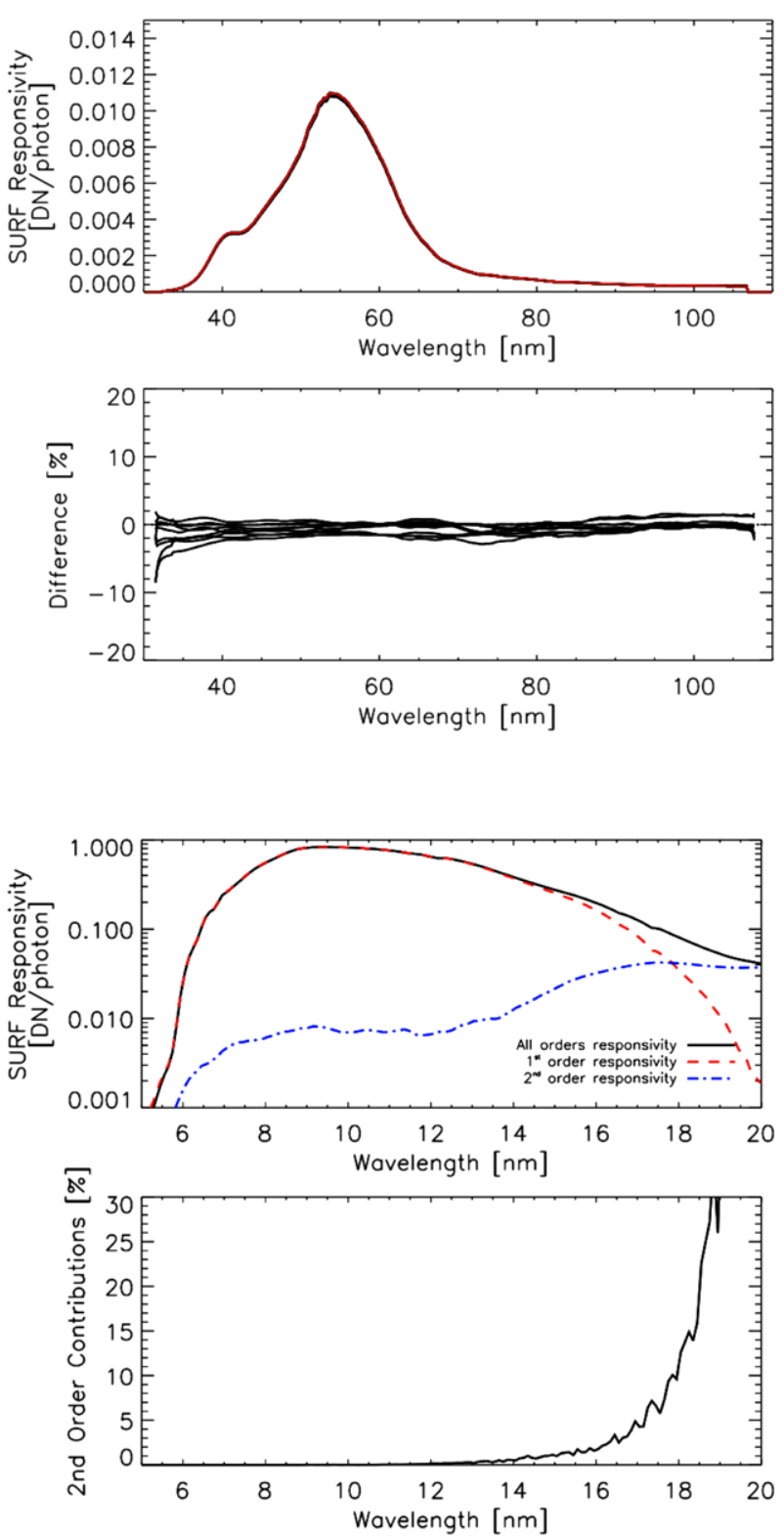

eliminating, or at least significantly reducing, the higher orders in these instruments using holographically ruled gratings that suppress even orders and in MEGS B using the dual orthogonal gratings.

The top panels of Figures 9 and 10 show the SURF responsivity without any correction (black), the true first-order responsivity (red), and the second-order responsivity (blue). These were determined using two beam energies. However, multiple combinations of beam energies were tested to verify the results. 
Figure 10 Results of the multiple-beam-energy method for MEGS A2. The top panel shows the SURF responsivity without any correction (black), the true first-order responsivity (red) and the second-order responsivity (blue). The bottom panel shows the expected contribution to the first-order spectrum from the second-order spectrum at half the wavelength.
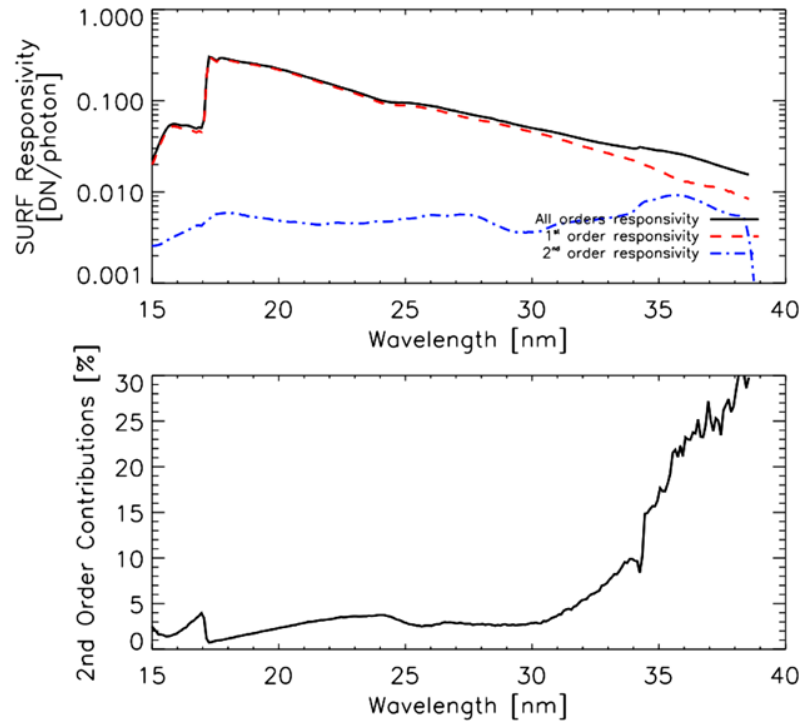

The bottom panels of Figures 9 and 10 show the expected contribution to the first-order spectrum from second order at half the wavelength:

$$
f_{2 \mathrm{nd}}=\frac{\frac{1}{2} R_{2}\left(\frac{\lambda}{2}\right)}{R_{1}(\lambda)} \times 100 \%
$$

For MEGS A1, there is very little contribution below $15 \mathrm{~nm}$. For MEGS A2, the higherorder contribution increases sharply near $34 \mathrm{~nm}$, which is the second-order rise due to the Al filter edge near $17 \mathrm{~nm}$.

\subsection{Flight Responsivity}

The final step in calibrating MEGS A and B is calculating the flight responsivity, defined in Equation (6). The irradiance from a solar MEGS image is the corrected count rate for that image divided by the flight responsivity, being the SURF response averaged over the solar disk field-of-view.

Figure 11 shows the flight responsivity for MEGS A1 and A2 (top) and the associated uncertainty (bottom), while Figure 12 shows the flight responsivity (top) and uncertainty (bottom) for MEGS B. The uncertainties are for individual pixels. For the final irradiance spectrum, pixels along the slit are added together into $0.02 \mathrm{~nm}$ wavelength bins, so the irradiance uncertainty is reduced when assuming random errors. Figures 13 and 14 show the ratio of the flight responsivity for the rocket MEGS instruments to the flight MEGS instruments. Both sets of MEGS instruments have similar responsivity. The MEGS A results might indicate a wavelength shift between these different calibrations; these possible wavelength shifts are expected to be resolved once solar measurements have been made. For MEGS B, the differences in responsivity are mostly related to different CCDs selected for the flight and rocket instruments. 
Figure 11 Flight responsivity for MEGS A1 and A2 (top) and the associated relative uncertainty (bottom).
Figure 12 Flight responsivity for MEGS B (top) and the associated relative uncertainty (bottom).
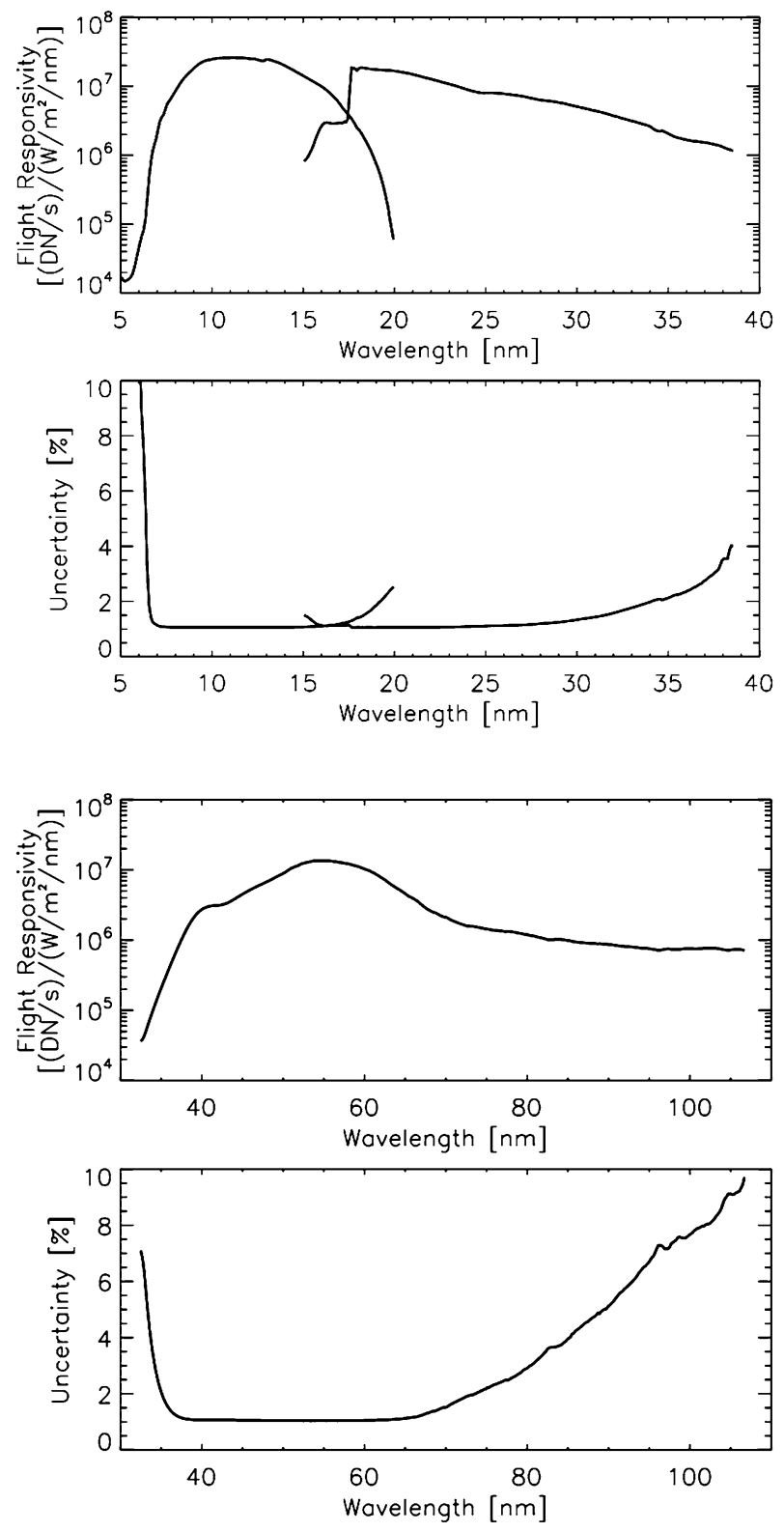

\section{MEGS P Calibration}

The MEGS P photometer is an integrated part of the MEGS B spectrograph and consists of a Si photodiode and Acton $122 \mathrm{~nm}$ filter located after the first MEGS B grating and positioned to measure the bright $\mathrm{H}$ I Lyman- $\alpha$ emission at $121.6 \mathrm{~nm}$. The MEGS P photodiode is not at the focal plane of this concave grating due to space limitations within the MEGS B housing. By not being at the focal plane, the spectrum across the diode is a fairly broadband of about $10 \mathrm{~nm}$. Even so, the Lyman- $\alpha$ emission still contributes about $98 \%$ of the total solar signal 
Figure 13 Responsivity for MEGS A1 and A2 (top) for flight and rocket instruments. We believe the differences are mostly due to a wavelength shift in the flight instrument. The bottom panel shows the percent differences between the flight responsivity for the rocket MEGS A channel and the flight MEGS A channel.

Figure 14 Responsivity for MEGS B (top) for flight and rocket instruments. We believe the differences are mostly due to variations in the CCDs. The bottom panel shows the percent differences between the flight responsivity for the rocket MEGS B channel and the flight MEGS B channel.
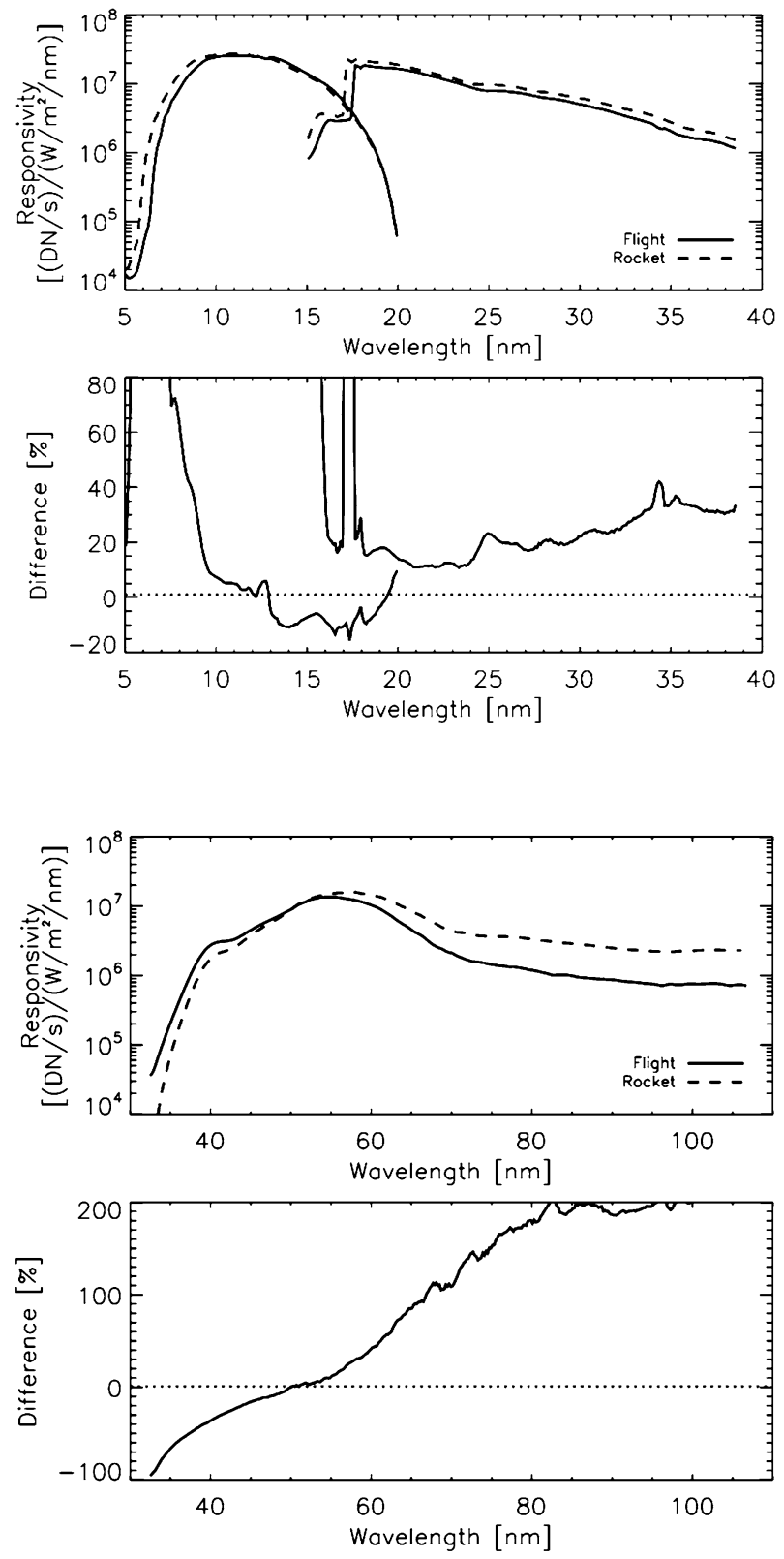

on the MEGS P photodiode. More details on the MEGS P instrument are given by Woods et al. (2010).

The MEGS P calibration uses a set of algorithms that draws on the heritage of many previous instruments using similar Si photodiodes with a bandpass filter as discussed in Section 3. This is also similar to the algorithms discussed by Didkovsky et al. (2010) for the ESP instrument on EVE. 


\subsection{MEGS P Algorithm}

The responsivity algorithm for MEGS $\mathrm{P}$ is a simplified version of that for MEGS A and B. As with MEGS A and $\mathrm{B}$, the raw signal is dark-corrected to get the corrected count rate $\left[C^{\prime}\right]$ :

$$
C^{\prime}(t)=\frac{C(t)-D(t)}{\Delta t}
$$

where $C$ is the data number $[\mathrm{DN}]$ from each integration from the photodiode electrometer that consists of a current amplifier and voltage-to-frequency converter [VFC]. The dark (background counts) is given by $D$, which is about $160 \mathrm{DN} \mathrm{second}^{-1}$ during SURF calibrations and ground testing. The dark correction is expected to be much more complicated in-flight due to radiation background effects, so an identical Si photodiode, but fully enclosed in an $\mathrm{Al}$ frame, is included as part of MEGS $\mathrm{P}$ to be a proxy of the background signal for the Lyman- $\alpha$ photodiode. The integration time $[\Delta t]$ is 0.25 seconds.

The SURF responsivity is then:

$$
R_{\mathrm{SURF}}\left(E_{\text {beam }}, \text { filter, } \alpha, \beta\right)=\frac{C^{\prime}\left(t, E_{\text {beam }}, \text { filter, } \alpha, \beta\right)}{I_{\mathrm{SURF}}(t) F_{\mathrm{SURF}}\left(E_{\text {beam }}, \alpha, \beta\right)}
$$

As with MEGS A and B, the corrected count rate is divided by the SURF beam current [ $\left.I_{\text {SURF }}\right]$ and the SURF beam flux $\left[F_{\text {SURF }}\right]$. The slit area of the MEGS B entrance slit is not included in the MEGS P responsivity as it is the same in the SURF calibration and solar observations.

The MEGS P responsivity needs to be corrected for the differences in the bandpass between SURF and solar measurements. As MEGS P is not in focus, it has a fairly broad bandpass and its responsivity is dependent on the spectrum being measured. The bandpasscorrected responsivity is given by:

$$
R_{\mathrm{P}}(\text { filter, } \alpha, \beta)=R_{\mathrm{SURF}}\left(E_{\text {beam }}, \text { filter, } \alpha, \beta\right) \frac{\Delta \lambda_{\text {Sun }}}{\Delta \lambda_{\text {SURF }}\left(E_{\text {beam }}\right)}
$$

where $\Delta \lambda_{\text {Sun }}$ is the bandpass of MEGS P for a solar spectrum, while $\Delta \lambda_{\text {SURF }}$ is the bandpass using SURF fluxes. These bandpasses are defined in Equations (18) and (19).

$$
\begin{aligned}
\Delta \lambda_{\mathrm{SURF}}\left(E_{\text {beam }}\right) & =\int_{\text {all } \lambda} \frac{F_{\mathrm{SURF}}\left(\lambda, E_{\text {beam }}\right)}{F_{\mathrm{SURF}}\left(121.6 \mathrm{~nm}, E_{\text {beam }}\right)} P_{\mathrm{G}}(\lambda) P_{\mathrm{F}}(\lambda) P_{\mathrm{D}}(\lambda) \mathrm{d} \lambda \\
\Delta \lambda_{\mathrm{Sun}} & =\int_{\text {all } \lambda} \frac{F_{\mathrm{Sun}}(\lambda)}{F_{\mathrm{Sun}}(121.6 \mathrm{~nm})} P_{\mathrm{G}}(\lambda) P_{\mathrm{F}}(\lambda) P_{\mathrm{D}}(\lambda) \mathrm{d} \lambda
\end{aligned}
$$

The bandpass is calculated by convolving the normalized spectrum, SURF flux, or the solar spectral irradiance, with the spectral profiles of the MEGS P optical components, which are the grating $\left[P_{\mathrm{G}}\right]$, filter $\left[P_{\mathrm{F}}\right]$, and diode $\left[P_{\mathrm{D}}\right]$. These profiles are not measured for MEGS P, but are estimated based on raytracing for the grating profile and from measurements on similar Acton filters and International Radiations Detectors (IRD) Si photodiodes. The detector profile is flat across the MEGS P range, but the filter and grating profile are strongly peaked near $121.6 \mathrm{~nm}$. These spectral profiles are normalized by their peak efficiencies, and the spectrum is normalized to its value at $121.6 \mathrm{~nm}$. Table 4 lists the spectral bandpasses for the different SURF beams and solar observations. As the SURF flux is a broad continuum, $\Delta \lambda_{\text {SURF }}$ is more representative of the full width of the MEGS P bandpass $(\approx 10 \mathrm{~nm})$. The solar spectrum is dominated by the H I Lyman- $\alpha$ emission, so the $\Delta \lambda_{\text {Sun }}$ is narrower $(\approx 1 \mathrm{~nm})$. 
Table 4 MEGS P spectral bandpass widths.

\begin{tabular}{lc}
\hline Irradiance source & Bandpass width $(\Delta \lambda)$ \\
\hline SURF 183 MeV & $10.641 \mathrm{~nm}$ \\
SURF 380 MeV & $10.618 \mathrm{~nm}$ \\
Sun-Solar-Cycle Min & $1.023 \mathrm{~nm}$ \\
Sun-Solar-Cycle Max & $1.016 \mathrm{~nm}$ \\
Sun - average & $1.020 \mathrm{~nm}$ \\
\hline
\end{tabular}

\subsection{Uncertainty Algorithm}

The uncertainty for $R_{\mathrm{P}}$ is derived from Equation (15) and is as follows.

$$
\sigma_{R_{\mathrm{P}}}^{2}=R_{\mathrm{P}}^{2}\left[\frac{\sigma_{C}^{2}+\sigma_{D}^{2}}{(C-D)^{2}}+\left(\frac{\sigma_{\Delta t}}{\Delta t}\right)^{2}+\left(\frac{\sigma_{F_{\mathrm{SURF}}}}{F_{\mathrm{SURF}}}\right)^{2}+\left(\frac{\sigma_{I_{\mathrm{SURF}}}}{I_{\mathrm{SURF}}}\right)^{2}+\left(\frac{\sigma_{\Delta \lambda_{\mathrm{SURF}}}}{\Delta \lambda_{\mathrm{SURF}}}\right)^{2}+\left(\frac{\sigma_{\Delta \lambda_{\mathrm{Sun}}}}{\Delta \lambda_{\mathrm{Sun}}}\right)^{2}\right]
$$

The relative uncertainties for $C, D, F_{\mathrm{SURF}}$, and $I_{\mathrm{SURF}}$ are each $1 \%$ or less. There is an unknown amount of uncertainty for the spectral bandpass (profile) at this time, but this could be the largest uncertainty for the MEGS P calibration. For now, the uncertainty of each spectral bandpass is assumed to be $10 \%$. Combining these as in Equation (16), the relative uncertainty for the MEGS P responsivity $\left[R_{\mathrm{P}}\right]$ is estimated to be $14 \%$.

\subsection{Calibration Results}

SURF calibration results for MEGS P are obtained simultaneously while doing the MEGS B calibrations, which primarily use $140 \mathrm{MeV}$ SURF beams. In addition, special MEGS P calibrations at $183 \mathrm{MeV}$ and at $380 \mathrm{MeV}$ are done at the center point $(\alpha=0, \beta=0)$ to minimize the MEGS P responsivity uncertainty. Using SURF beam energy of $183 \mathrm{MeV}$ and a beam current of $300 \mathrm{~mA}$, the quantity $C^{\prime}$ in Equation (15) is about $150 \mathrm{DN}$ second ${ }^{-1}$. The center point $R_{\mathrm{P}}$ is $1421 \mathrm{DN}\left(\mathrm{W} \mathrm{m}^{-2}\right)^{-1}$ for the flight $\mathrm{EVE}$ and is $1922 \mathrm{DN}\left(\mathrm{W} \mathrm{m}^{-2}\right)^{-1}$ for the protoflight (rocket) EVE, each having relative uncertainty of about $14 \%$. The largest contribution to the uncertainty is the bandpass uncertainty. A future improvement for MEGS P calibrations is to measure the bandpass directly instead of estimating the bandpass by analysis.

The variation of the responsivity across the solar FOV $\left(0.5^{\circ}\right)$ is less than $1 \%$ and has a sensitivity of about $0.02 \%$ per arcminute. With solar pointing from SDO expected to vary much less than one arcminute, a FOV correction is not currently included in the MEGS P solar-irradiance algorithm.

\subsection{Application for MEGS In-flight Calibrations}

In addition to the scientific reasons for measuring Lyman- $\alpha$, another purpose for the MEGS $\mathrm{P}$ instrument is to be a calibration proxy for other $\mathrm{H}$ and $\mathrm{He}$ emission lines in the MEGS A and B spectra. In particular, if MEGS P has little degradation, which is expected to be less than $1 \%$ per year, then the MEGS $\mathrm{P}$ can serve as reference proxy for degradation of MEGS A and B. From TIMED/SEE observations, the relationship of the H I Lyman- $\alpha$ (121.6 nm) emission to $\mathrm{H} \mathrm{I}$ emission lines and continuum as well as He I and He II emission 
Figure 15 Contrast ratios for $\mathrm{HI}, \mathrm{He}$, and He II emissions relative to $\mathrm{H}$ I Lyman- $\alpha$ emission. The black triangles are the short-term contrast ratios, and the green diamonds are the long-term contrast ratios, both derived using TIMED/SEE Level 3 data. The red plus symbols are the contrast ratios in the SC21REFW solar reference spectrum/model (Hinteregger, Fukui, and Gilson, 1981).

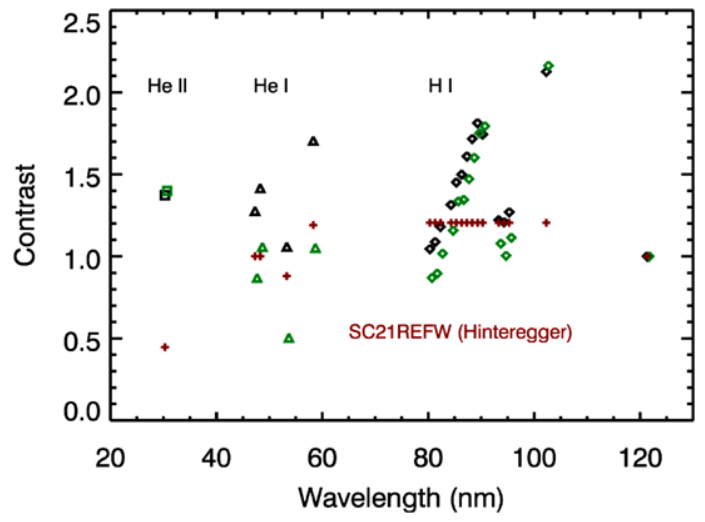

lines in the EUV are all accurately known. The contrast ratio, being the ratio of variability between one emission and a proxy, is expected to be the same for short-term variability ( $<81$ days) and long-term variability ( $>81$ days) if the emission and the proxy are from the same solar atmospheric layer (Woods et al., 2000). This expectation was established for the solar far ultraviolet irradiances from Upper Atmosphere Research Satellite (UARS)/SOLar STellar Irradiance Comparison Experiment (SOLSTICE).

To see if this relationship holds for the EUV wavelengths, the comparison of short-term and long-term variability is shown in Figure 15 using SEE data. As expected, the short-term and long-term contrast ratios are about the same for the H I, He I, and He II emissions; however, there are larger differences for the He I emissions. The uncertainty in the SEE degradation trends in the 45 to $70 \mathrm{~nm}$ range appear to be the cause of this difference for the long-term contrast ratios for the He I emissions. The short-term contrast ratios are much more accurate than the long-term contrast ratios because instrument degradation over 81 days is much less of a concern than that over six years. Therefore, the short-term contrast ratios, as shown in Figure 15 and listed in Table 5, will be used with the MEGS P solar Lyman- $\alpha$ measurements to help establish the degradation trends of MEGS A and B. These contrast ratios are derived using the SEE Level 3 (Version 10) data given in 1-nm intervals. The underflight rocket EVE calibrations provide the ultimate long-term accuracy, so the MEGS P proxy estimates for the $\mathrm{H}$ and $\mathrm{He}$ emissions between 25 and $103 \mathrm{~nm}$ are intended only for the time between the calibration rocket flights.

An interesting result from generating these contrast ratios for the $\mathrm{H}$ and $\mathrm{He}$ emissions in the EUV relative to the solar Lyman- $\alpha$ is that the contrast ratios are not constant values as shown for the Hinteregger, Fukui, and Gilson (1981) results (proxy model). The SEE data are in 1-nm intervals, so contamination of a few weaker lines is only slightly affecting these results, but there are systematic trends, notably in the H I continuum, that likely indicate intrinsic solar-variability relationships. Existing solar empirical models that use the Hinteregger, Fukui, and Gilson (1981) results should be updated with the TIMED/SEE results. Because these relationships are based on short-term variations, we can independently verify them during the first year of the SDO mission.

\section{MEGS SAM Calibration}

The Solar Aspect Monitor (SAM) instrument is a simple pinhole-camera optical design that provides a low-resolution $\left(\approx 15\right.$ arcseconds pixel $\left.^{-1}\right)$ solar image onto an unused part of the 
Table 5 Contrast ratios for H I, He I, and He II emissions relative to MEGS P Lyman- $\alpha$.

\begin{tabular}{|c|c|c|c|c|c|}
\hline Emission & Wavelength (nm) & $\begin{array}{l}\text { Short-term } \\
\text { contrast ratio }\end{array}$ & Emission & Wavelength (nm) & $\begin{array}{l}\text { Short-term } \\
\text { contrast ratio }\end{array}$ \\
\hline $\mathrm{H} \mathrm{I}$ & 80.5 & 1.045 & He I & 47.5 & 1.277 \\
\hline $\mathrm{H} \mathrm{I}$ & 81.5 & 1.088 & $\mathrm{He} \mathrm{I}$ & 48.5 & 1.416 \\
\hline H I & 82.5 & 1.180 & He I & 53.7 & 1.059 \\
\hline $\mathrm{H} \mathrm{I}$ & 84.5 & 1.315 & $\mathrm{He} \mathrm{I}$ & 58.4 & 1.706 \\
\hline $\mathrm{H} \mathrm{I}$ & 85.5 & 1.451 & He II & 30.4 & 1.373 \\
\hline
\end{tabular}

\begin{tabular}{lrl} 
H I & 87.5 & 1.610 \\
H I & 88.5 & 1.717 \\
H I & 89.5 & 1.813 \\
H I & 90.5 & 1.743 \\
H I Ly- $\varepsilon$ & 93.8 & 1.221 \\
H I Ly- $\delta$ & 95.0 & 1.239 \\
H I Ly- $\beta$ & 102.6 & 2.126 \\
\hline
\end{tabular}

MEGS A CCD. SAM has two operating modes depending on which filter is used. The first uses a visible-light filter for alignment purposes for both ground and in-flight calibrations to produce better than one-arcminute alignment (pointing) information for the MEGS spectrographs. The second mode uses an X-ray filter, equivalent to the ESP zeroth-order filter consisting of mostly $\mathrm{Al}$ and $\mathrm{Ti}$, to provide solar images and solar spectra for the 0.1 to $7 \mathrm{~nm}$ range. The X-ray spectrum is possible because the filter and pinhole size are designed so that only single photon events can be detected in a ten-second integration in any given pixel. For the Si-based CCD, each X-ray photon produces a few hundred electron-hole pairs within a couple pixels. The number of electrons is expected to be the energy of the photon divided by the Si band-gap energy $(3.65 \mathrm{eV})$. This technique is advantageous because not only is an image produced but also the energy of each photon event, and therefore the photon wavelength, can be determined. The current plan for SAM data processing with the X-ray filter is to produce a spectrum in 1-nm intervals between 0.1 and $7 \mathrm{~nm}$ with a cadence of one minute and an X-ray image with a cadence of five minutes. More information about SAM and MEGS A CCD is provided by Woods et al. (2010).

The solar aspect mode (visible-light filter) will be used only once a day to verify the pointing of the MEGS spectrographs, while the X-ray filter will be used most of the time as part of SAM's normal science observations. The visible solar images are dominated by diffraction, as the pinhole size has only a 26 micron diameter, and thus are more useful for solar position information than for scientific research. The solar diameter and diffraction pattern are estimated to provide a Sun-center position with an accuracy to better than two pixels ( 0.5 arcminute). The diffraction is not an issue with X-ray photons so the X-ray solar images are useful for scientific research. Active regions are expected to dominate the X-ray images. That is, these images do not have a uniform disk, so solar position from an X-ray image is more challenging and thus they have less accuracy than the visible images.

The following discussion for SAM concerns only the calibrations and processing for the X-ray filter. While the photon-detection technique is known to work in the X-rays for $\mathrm{Si}$ detectors, there is no heritage for processing solar X-ray data from LASP's previous satellite instrument programs. The prototype EVE instrument did fly on a sounding-rocket flight in 
April 2008, so there are some limited solar data during solar-cycle minimum conditions to validate the SAM processing algorithms prior to the SDO launch.

\subsection{Responsivity Algorithm}

The responsivity algorithm for SAM is similar to that of MEGS A, B, and P and is given by:

$$
R_{\mathrm{SURF}}(i, j, \lambda)=\frac{\sum_{\text {all images }} \frac{N_{\text {photons }}\left(i, j, t, \lambda, E_{\text {beam }}\right)}{I_{\mathrm{SURF}}(t)}}{F_{\mathrm{SURF}}\left(\lambda, E_{\text {beam }}\right) \Delta t A_{\text {slit }} \Delta \lambda}
$$

Here, $N_{\text {photons }}$ is the number of photon events at a wavelength $\lambda$ within a band $\Delta \lambda$. To improve statistics, events from many images are first normalized by the SURF beam current and then added together. In order to compare the number of photon events measured with the SURF signal, the SURF flux is multiplied by the integration time $[\Delta t]$. The slit area $\left[A_{\text {slit }}\right]$ is the area of the SAM entrance slit $\left(5.31 \times 10^{-10} \mathrm{~cm}^{2}\right)$. The responsivity $\left[R_{\mathrm{SURF}}\right]$ consists of the transmission of the $\mathrm{Ti}-\mathrm{Al}-\mathrm{C}$ filter and the quantum throughput [QT] of the CCD. With the CCD QT being almost $100 \%$, the responsivity result is primarily the spectral shape of the filter's transmission.

Each photon event is assumed to be an isolated event. Double or more concurrent and co-located events, which are intended by design to be less than $10 \%$ of the time for solar maximum conditions, will effectively be treated incorrectly as a single photon event but with more energy (shorter wavelength). The algorithm to detect a photon event starts by using a dark- and particle hit-corrected MEGS A image, similar to Equation (1). Then the algorithm identifies each pixel within the SAM image area that has a signal of greater than ten $\mathrm{DN}$ ( $\approx 20$ electrons). The surrounding pixels with a signal greater than two DN are added together to get the energy of the photon event in DN. This number is then converted to energy units by multiplying by the gain $\left(G_{\mathrm{SAM}}\right.$, about two electrons per DN) and the Si band-gap energy $\left(E_{\mathrm{Si}}, 3.65 \mathrm{eV}\right.$ per electron-hole pair). If there are two photon events adjacent to each other, the algorithm splits the signal of the intermediate pixel between the two events. Events that are larger than $3 \times 3$ pixels are assumed to contain multiple photon events and are not included in the spectrum, although the final spectrum is adjusted to reflect the estimated number of events lost in this manner.

Binning by wavelength does not work well for SAM spectra, as a single DN may represent multiple wavelength bins at longer wavelengths or a single wavelength bin may include several DN at shorter wavelengths. Therefore, the SAM spectrum is accumulated in energy bins, with each bin equivalent to one DN. In order to create an image, the pixel location of photons events is recorded and these photon events are accumulated into an image over several integrations. With a long enough accumulation period (several minutes to an hour or more), one could separate the X-ray images into multiple wavelength bands within the 0.1 to $7 \mathrm{~nm}$ range.

Although the sounding-rocket flight demonstrated the effectiveness of this single-photonevent method for the use with solar data, the SURF calibrations could not properly count enough single photon events for this method to provide accurate results. This shortcoming for SURF calibrations is due to the SURF beam's small size, which results in the image being spread over only a couple of pixels (versus hundreds of pixels for a solar image). As a result, all of the photon events are concentrated in a few pixels, and multiple events are mistakenly counted as higher-energy single events. 
Therefore, in order to properly calibrate SAM, it was necessary to instead use Equations $(22-24)$. The measured signal $\left[S_{\text {measured }}\right]$ in $\mathrm{eV}$ second ${ }^{-1}$ is given by:

$$
S_{\text {measured }}\left(t, E_{\text {beam }}\right)=\sum_{i, j} \frac{C_{\text {image }}\left(i, j, t, E_{\text {beam }}\right)}{\Delta t} G_{\mathrm{SAM}} E_{\mathrm{Si}}
$$

where $C_{\text {image }}$ is the number of $\mathrm{DN}$ in each pixel, $G_{\mathrm{SAM}}$ the gain to convert $\mathrm{DN}$ to electrons, and $E_{\mathrm{Si}}$ is energy conversion from electrons to $\mathrm{eV}$ (3.65 eV for silicon). The predicted signal $\left[S_{\text {predicted }}\right]$ is:

$$
S_{\text {predicted }}\left(t, E_{\text {beam }}\right)=\int_{0}^{\infty} R_{\mathrm{SAM}}(\lambda) F_{\mathrm{SURF}}\left(\lambda, E_{\text {beam }}\right) I_{\mathrm{SURF}}(t) A_{\text {slit }} \mathrm{d} \lambda
$$

The responsivity of SAM $\left[R_{\mathrm{SAM}}\right]$ is the transmission model of the $\mathrm{Ti}-\mathrm{Al}-\mathrm{C}$ filter and the CCD detector based on the atomic parameters from Henke, Gullikson, and Davis (1993). If the SAM responsivity is correct, we expect the ratio of $S_{\text {measured }}$ to $S_{\text {predicted }}$ to be unity. This ratio represents a scaling factor $\left[f_{\mathrm{SAM}}\right]$ on how to correct the SAM responsivity.

$$
f_{\text {SAM }}\left(E_{\text {beam }}\right)=\frac{S_{\text {measured }}\left(t, E_{\text {beam }}\right)}{S_{\text {predicted }}\left(t, E_{\text {beam }}\right)}
$$

By using multiple beam energies $(331,361,380$, and $408 \mathrm{MeV})$, the different scaling factors from each beam energy indicate roughly how the responsivity needs to be adjusted in wavelength. The filter thicknesses were adjusted in the transmission model until $f_{\text {SAM }}$ was near unity.

The value of the SAM gain factor $\left[G_{\text {SAM }}\right]$ was determined by using several methods. The primary method involved comparing the solar spectrum created from the SAM data when assuming a gain value of two with the Whole Heliosphere Interval (WHI) solar spectrum generated by SORCE/XPS (Woods et al., 2009). A distinct peak in irradiance occurred in both spectra, but the SAM spectrum had to be shifted to higher energy by a factor of about 1.3 in order for these peaks to occur at the same wavelength. This finding suggests that the SAM gain factor should have a value of $2.67 \mathrm{e}^{-} \mathrm{DN}^{-1}$. This gain factor, however, only applies to the single-photon-event method used for solar analysis, and does not apply to the signal integration method because it only registers signals greater than two DN. To get the correct gain for SURF calibrations, the signal was extrapolated to determine what the signal would theoretically be if the limit were set at zero DN. Doing this, the signal would be 7\% greater, so the gain value used for SURF calibrations, should be $7 \%$ less than for the solar calibrations, yielding a value of $2.47 \mathrm{e}^{-} \mathrm{DN}^{-1}$.

The spectral-binning method takes advantage of the presence of several solar spectrum lines from the MEGS A2 slit that appear next to the SAM image. The wavelengths of these spectral lines are known, so therefore the energies of the photon events in these lines are also known. Therefore, the gain can be directly derived from the ratio of the expected photon signal from a specific wavelength to the measured photon signal. This method generates a gain value of $3.1 \pm 0.44 \mathrm{e}^{-} \mathrm{DN}^{-1}$. On account of the large uncertainty of this method, we do not use this value for the gain (using instead the $2.47 \mathrm{e}^{-} \mathrm{DN}^{-1}$ from the other method); however, this method does provide limited validation for the other SAM gain values we calculated.

In order to reflect the actual resolution possible with SAM, we smoothed the responsivity model based on the resolution calculated by Equation (25) at each energy before applying the model to the solar data. Again, $G_{\mathrm{SAM}}$ is the gain factor, while $D_{\mathrm{S}}$ is the dark correction 
for the CCD image, and $F$ is the Fano factor, which has a value of 0.115 for silicon. $h c / \lambda$ converts wavelength of the photon into energy, while $1 / E_{\mathrm{Si}}$ converts energy to number of electron-hole pairs. We have

$$
\Delta E=8.61 \sqrt{\left(G_{\mathrm{SAM}} \sqrt{D_{\mathrm{S}}}\right)^{2}+\left(\frac{h c}{\lambda} \times \frac{F}{E_{\mathrm{Si}}}\right)}
$$

While the SAM X-ray algorithms are developed, they require significantly more validation. This SAM technique for X-ray spectra and images is at an experimental stage. Much can be learned from a longer time series of solar observations than what is possible over a single sounding rocket flight. The SAM results are not a required part of the EVE results, as the primary source for the 0.1 to $7 \mathrm{~nm}$ band is the ESP zeroth-order channel that has the same spectral bandpass as SAM. If the SAM algorithm is found to be properly providing results consistent with the ESP results, then the longer-term degradation for SAM (filter transmission and detector QT) can be tracked with the ESP results. Of particular concern with SAM is the double or more photon events that can contaminate the SAM spectra and images, and how this contamination might evolve with solar activity (cycle minimum to maximum and during flares) and with variation in the number of active regions on the solar disk.

\subsection{Uncertainty Algorithm}

The uncertainty for the SAM responsivity $\left[R_{\mathrm{SAM}}\right]$ is derived from Equations $(22-24)$ and is as follows:

$$
\begin{aligned}
\sigma_{R_{\mathrm{SAM}}}^{2}= & R_{\mathrm{SAM}}^{2}\left[\left(\frac{\sigma_{C_{\text {image }}}}{C_{\text {image }}}\right)^{2}+\left(\frac{\sigma_{\Delta t}}{\Delta t}\right)^{2}+\left(\frac{\sigma_{G_{\mathrm{SAM}}}}{G_{\mathrm{SAM}}}\right)^{2}+\left(\frac{\sigma_{F_{\mathrm{SURF}}}}{F_{\mathrm{SURF}}}\right)^{2}+\left(\frac{\sigma_{I_{\mathrm{SURF}}}}{I_{\mathrm{SURF}}}\right)^{2}\right. \\
& \left.+\left(\frac{\sigma_{f_{\mathrm{SAM}}}}{f_{\mathrm{SAM}}}\right)^{2}\right]
\end{aligned}
$$

As SAM counts photons, the uncertainty for $C_{\text {image }}$ is estimated as the square root of $C_{\text {image }}$. The uncertainty for the $G_{\mathrm{SAM}}$ is about $10 \%$. The relative uncertainties for SURF flux [ $F_{\mathrm{SURF}}$ ] and beam current $\left[I_{\mathrm{SURF}}\right]$ are both $1.0 \%$. The uncertainty of the scaling factor $\left[\sigma_{f}\right]$ is 0.084 . The combination of these uncertainties yields a relative uncertainty of $16 \%$ for SAM responsivity.

\subsection{Calibration Results}

As mentioned in Section 7.1, the SURF calibrations for SAM did not provide reliable photon events for using Equation (19). Therefore, the SAM responsivity is based on the integrated signal from the SURF calibrations as described by Equations $(22-24)$. In addition to the data from the calibrations performed at SURF in August 2007, data from a previous SURF calibration in February 2007 and well as rocket calibrations from October 2007 and January 2009 contributed to the calibration process. The data were analyzed using Equation (22) in order to calculate a scaling factor $\left[f_{\mathrm{SAM}}\right]$. The responsivity model was then adjusted by altering the filter thicknesses to achieve values for $f_{\mathrm{SAM}}$ close to unity. As shown in Table 6 , the adjusted responsivity model generated $f_{\text {SAM }}$ values that are within $20 \%$ of the desired value. The mean final scaling factor is 0.97 with a standard deviation of 0.084 . The consistency of the results between the 2007 and 2009 calibrations indicates that SAM did 
Table 6 Scaling factor results for SAM calibration sets. Results are based on the calibrated responsivity model and represent different beam energies. Instances where lower currents had to be used due to lack of data at a higher current are marked with an asterisk $(*)$.

\begin{tabular}{lllll}
\hline Calibration set & $\begin{array}{l}\text { Beam energy } \\
(\mathrm{MeV})\end{array}$ & $\begin{array}{l}\text { Beam } \\
\text { current }(\mathrm{mA})\end{array}$ & $\begin{array}{l}\text { Original scaling } \\
\text { factor }\end{array}$ & $\begin{array}{l}\text { Final scaling } \\
\text { factor }\end{array}$ \\
\hline $\begin{array}{l}\text { Flight instrument } \\
\text { (February 2007) }\end{array}$ & 408 & $1.6 \times 10^{-4}$ & 0.7602 & 1.0747 \\
& 380 & $1.8 \times 10^{-4}$ & 0.6934 & 1.0389 \\
& 361 & $2.0 \times 10^{-4}$ & 0.6185 & 0.9615 \\
& $331^{*}$ & $1.0 \times 10^{-4}$ & 0.4769 & 0.7819 \\
Flight instrument & 408 & $1.6 \times 10^{-4}$ & 0.7742 & 1.0945 \\
(August 2007) & 380 & $1.8 \times 10^{-4}$ & 0.6796 & 1.0181 \\
& 361 & $2.0 \times 10^{-4}$ & 0.6289 & 0.9777 \\
& $331^{*}$ & $1.0 \times 10^{-4}$ & 0.5416 & 0.8852 \\
Rocket pre-flight & 408 & $2.0 \times 10^{-4}$ & 0.7545 & 1.0667 \\
(October 2007) & 380 & $2.0 \times 10^{-4}$ & 0.6519 & 0.9767 \\
& 361 & $2.0 \times 10^{-4}$ & 0.6256 & 0.9726 \\
& 331 & $2.0 \times 10^{-4}$ & 0.5511 & 0.9036 \\
Rocket post-flight & $408^{*}$ & $2.0 \times 10^{-5}$ & 0.6844 & 0.9675 \\
(January 2009) & 380 & $2.0 \times 10^{-4}$ & 0.6824 & 1.0224 \\
& $361^{*}$ & $2.0 \times 10^{-5}$ & 0.5556 & 0.8637 \\
& 331 & $2.0 \times 10^{-4}$ & 0.5616 & 0.9208 \\
\hline
\end{tabular}

not degrade much, if at all, over two years. The SURF calibration results from the flight and rocket instruments also agree remarkably well with each other. Using Equation (26), the average uncertainty of the SAM responsivity was found to be $7.5 \%$ for the main SAM wavelength range $(0.1-7 \mathrm{~nm})$.

The initial values for the SAM responsivity calculation were $C$ thickness of $40 \mathrm{~nm}, \mathrm{Al}$ thickness of $100 \mathrm{~nm}$, Ti thickness of $300 \mathrm{~nm}, \mathrm{SiO}$ thickness of $7 \mathrm{~nm}$, and Si detector thickness of five microns. The scaling factors have a systematic variation of 0.44 for $331 \mathrm{MeV}$ beam to 0.60 for $408 \mathrm{MeV}$ beam. After the filter thicknesses were adjusted so that the scaling factors were close to unity, the thicknesses were $80 \mathrm{~nm}$ for C, $200 \mathrm{~nm}$ for Al, and $320 \mathrm{~nm}$ for Ti. The thickness of the Si detector remained five microns. The calculated responsivity with these parameters is consistent for both the flight and rocket SAM instruments. Figure 16 shows the SAM responsivity as determined from the SURF calibrations. The SAM responsivity, as expected, has the appearance of the $\mathrm{Ti}-\mathrm{Al}-\mathrm{C}$ filter transmission profile. The $\mathrm{Ti}$ part of the filter is expected to have a dip in transmission near $2.5 \mathrm{~nm}$ and this dip is used as a wavelength calibration for SAM calibrations. Triplett et al. (2007) provide additional information about filter calibrations performed prior to the SURF calibrations.

\section{Solar EUV Measurements on 14 April 2008}

The long-term degradation correction for the flight EVE depends on cross-calibrations with the prototype EVE flown on annual sounding rocket flights. This prototype experiment is calibrated using the same techniques and algorithms described in this paper, and the calibration rocket measurement provides a new reference that can be used to adjust the flight 
Figure 16 SAM Transmission. This transmission includes the transmission of the foil filter and absorption of the Si CCD (calculated as one minus the $\mathrm{Si}$ transmission). These transmissions are calculated using the Henke, Gullikson, and Davis (1993) atomic constants, and the thickness of the filter materials were adjusted to agree with SURF calibration results.

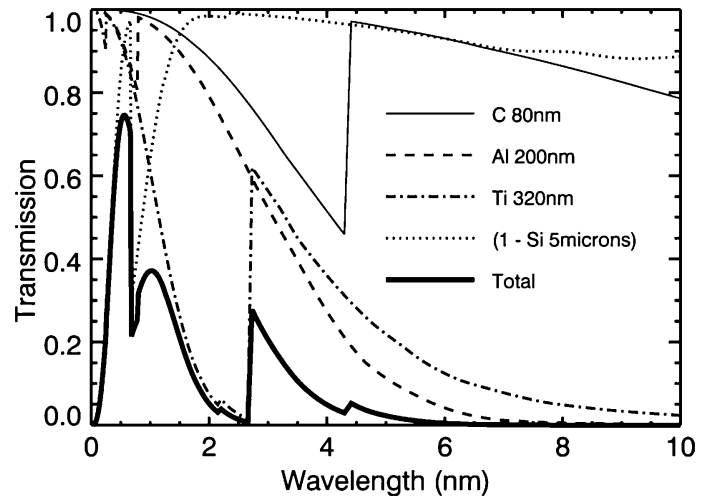

EVE results. Both pre- and post-flight calibrations of the rocket instruments are performed to measure the degradation, if any, of the rocket instruments. This rocket payload for EVE was built and launched to provide the final calibration underflight for the TIMED/SEE instrument on 14 April 2008 from the White Sands Missile Range in New Mexico. The initial results for the solar EUV irradiance measured during this flight were presented by Chamberlin et al. (2009), but these results did not include the post-flight calibration where significant improvements were made and applied. The more accurate results from MEGS A and B observations, which include the post-flight SURF calibration, are presented here, along with the first results from MEGS P and SAM. These rocket measurements have been used to update the TIMED/SEE degradation functions (included in SEE Version 10 data products), to provide a reference spectrum for solar-cycle minimum conditions (Chamberlin et al., 2009; Woods et al., 2009), and to improve upon the flight EVE data-processing system.

\subsection{MEGS A and B}

The calibrated MEGS A1, A2, and B spectra from the sounding-rocket flight are shown in Figure 17, along with the uncertainty of the solar irradiance. These results are very similar to those presented by Chamberlin et al. (2009). This latest irradiance result with the prototype EVE includes the post-flight calibrations, which provided improvements especially for the higher-order corrections. No significant degradation was found between the pre- and post-flight calibrations. The main difference between this spectrum and that reported by Chamberlin et al. (2009) is in the 35 to $45 \mathrm{~nm}$ range where the new results have higher irradiance. This wavelength range is improved now with more accurate, higher-order corrections for MEGS A2 and improved calibrations for MEGS B. The other range of notable difference is 65 to $105 \mathrm{~nm}$, which has slightly higher irradiance than the Chamberlin et al. (2009) results.

\subsection{MEGS P}

MEGS P provides a measurement of the H I Lyman- $\alpha$ emission at $121.6 \mathrm{~nm}$. The April 2008 flight had a few minutes of solar observations, and it was during solar minimum conditions. The data are averaged over the flight at altitudes greater than $250 \mathrm{~km}$ so that the contribution from atmospheric absorption is not significant. The MEGS P irradiance $[I]$ is calculated from:

$$
I(121.6 \mathrm{~nm})=\frac{C^{\prime}(t, \text { filter })-C_{\mathrm{vis}}(t)}{R_{\mathrm{P}}} f_{\text {degrad }} f_{1 \mathrm{AU}}
$$



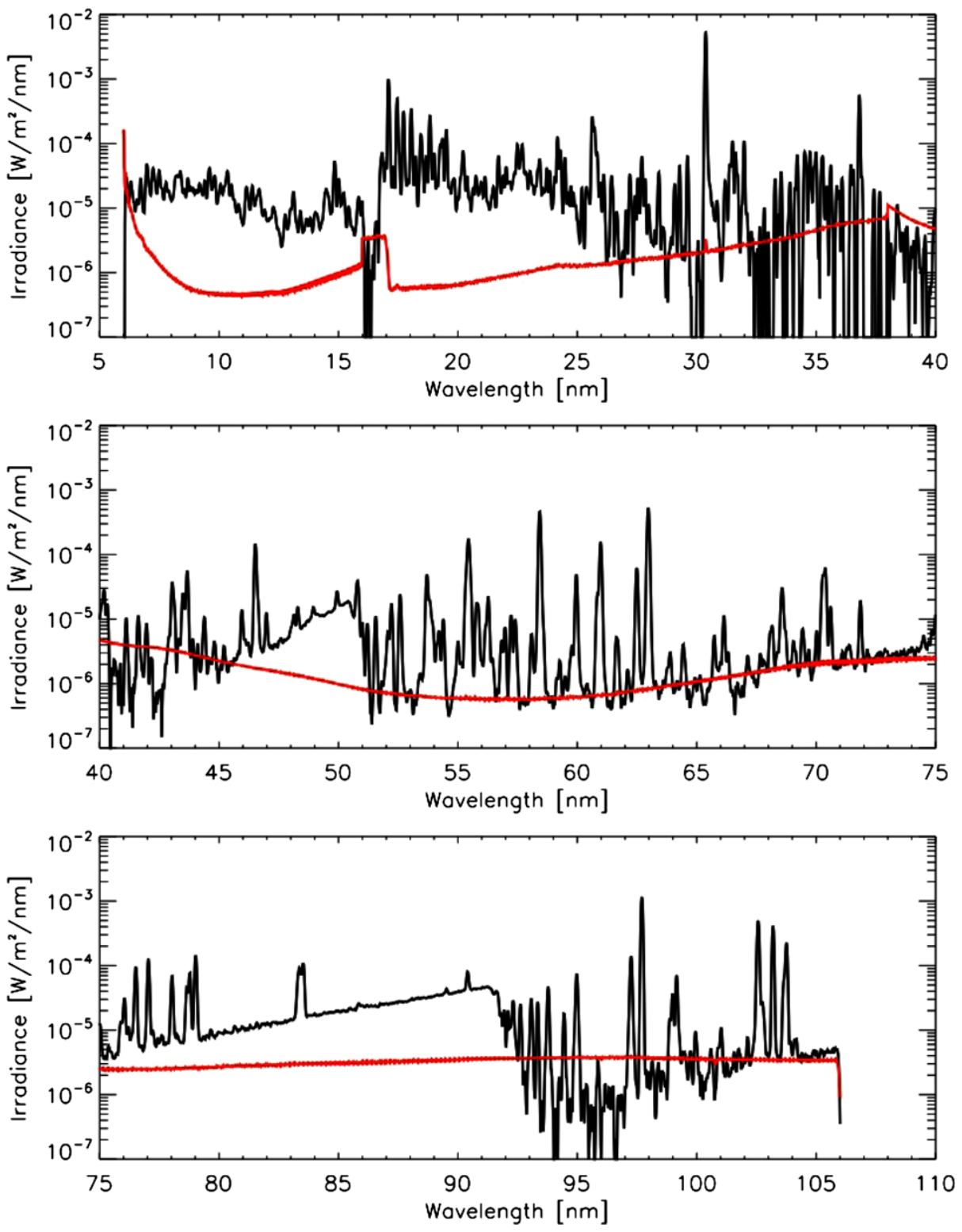

Figure 17 MEGS A1, A2, and B spectra for 14 April 2008. The uncertainty for the solar irradiance is also shown (red line).

where $C^{\prime}$ [Equation (15)] is the corrected count rate for solar data, $C_{\text {vis }}$ is the visible-light correction, $R_{\mathrm{P}}$ is the responsivity defined by Equation (17), $f_{\text {degrad }}$ is the degradation correction, and $f_{1 \mathrm{AU}}$ is the correction to normalize the irradiance to $1 \mathrm{AU}$. From SURF calibration analysis and the 2008 flight, the visible-light correction is not necessary $\left(C_{\text {vis }}=0.0\right)$, but it will be further validated with in-flight measurements after SDO is launched. 

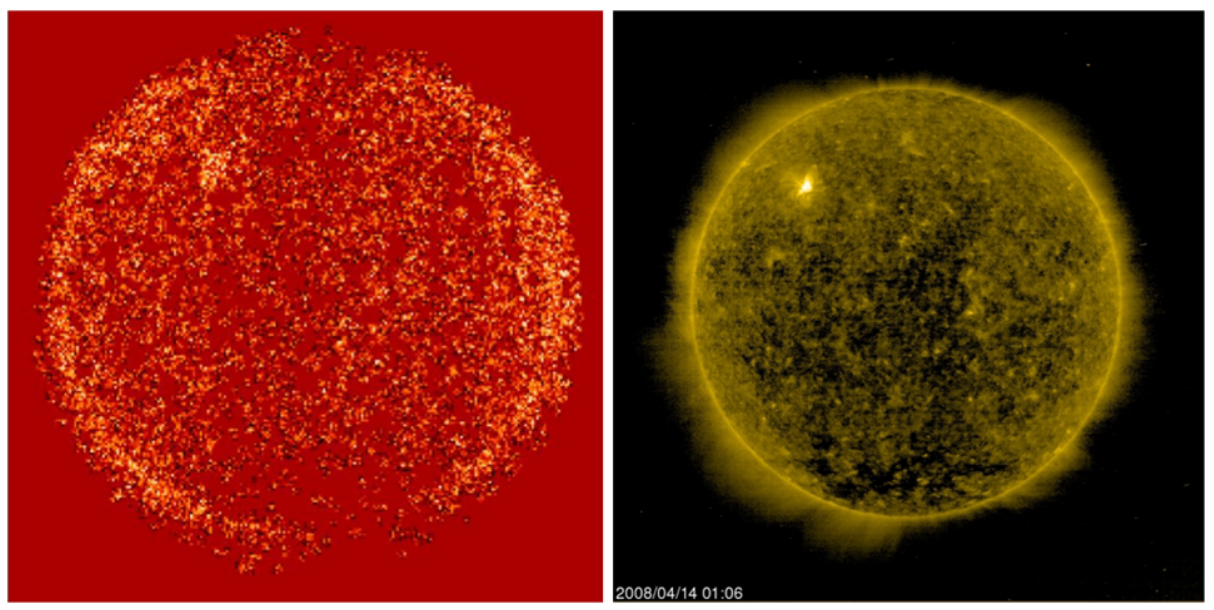

Figure 18 Solar X-ray image from SAM (left) and SOHO/EIT $28.4 \mathrm{~nm}$ image (right), courtesy NASA/ESA $\mathrm{SOHO} / \mathrm{EIT}$ consortium. Both images are representative of the solar corona. The SAM image clearly shows the small active region and coronal emissions above the limb of the Sun.

The MEGS P signal, corrected for dark counts, is $30 \mathrm{DN}$ second ${ }^{-1}$ for this rocket measurement. The irradiance result is $0.0039 \mathrm{~W} \mathrm{~m}^{-2}$, which is $30 \%$ less than the SORCE/ SOLSTICE 121 to $122 \mathrm{~nm}$ irradiance result of $0.0057 \mathrm{~W} \mathrm{~m}^{-2}$. This result only uses postflight MEGS calibrations in January 2009 (pre-flight rocket MEGS-P calibrations were not obtained). It seems unlikely that MEGS P degraded after the rocket flight; therefore, we suspect that the spectral bandpass (profile) for MEGS P is not accurate. The profile is strongly dependent on the Acton Lyman- $\alpha$ filter profile and also the spectral bandpass from the MEGS P first grating; neither is measured directly for MEGS P. Decreasing the $\Delta \lambda_{\text {Sun }}$ and/or increasing the $\Delta \lambda_{\text {SURF }}$ could bring the rocket MEGS P result into better agreement with SOLSTICE. If the flight MEGS P also measures lower irradiance than SOLSTICE, then we will assume the MEGS P spectral bandpass needs to be adjusted to be in agreement with SOLSTICE.

\subsection{SAM}

Only a few photon events were expected to be recorded during the rocket flight as the flight was during solar minimum. There was one small active region that appeared the day prior to launch and it was located in the northeast quadrant of the observable solar disk. The SAM image is presented in Figure 18, and this image is a compilation of all 17 ten-second images that were obtained during the rocket flight. This image shows the aforementioned active region, as well as the solar corona above the limb of the Sun. Also shown for comparison is the SOHO/EIT image for the day, which has much higher spatial resolution. The SAM image is representative of the coronal bremsstrahlung continuum and emission lines in the 0.1 to $7 \mathrm{~nm}$ band, and the EIT $28.4 \mathrm{~nm}$ image measures the bright, coronal Fe xv emission line and several other weaker emission lines near $28.4 \mathrm{~nm}$.

As mentioned, the advantage of SAM is that the individual photon events provide the energy, and thus the wavelength, for each photon. Once all of the photon events are processed, a solar spectrum can be produced for the rocket flight, and this spectrum is shown in Figure 19. The WHI spectrum generated by fitting model spectra to XPS broadband measurements is also shown for comparison (Woods et al., 2009). 
Figure 19 SAM solar spectrum from the April 2008 rocket flight. The SAM spectrum is shown as a thick line. The WHI reference spectrum from XPS is shown for comparison as a thin line.

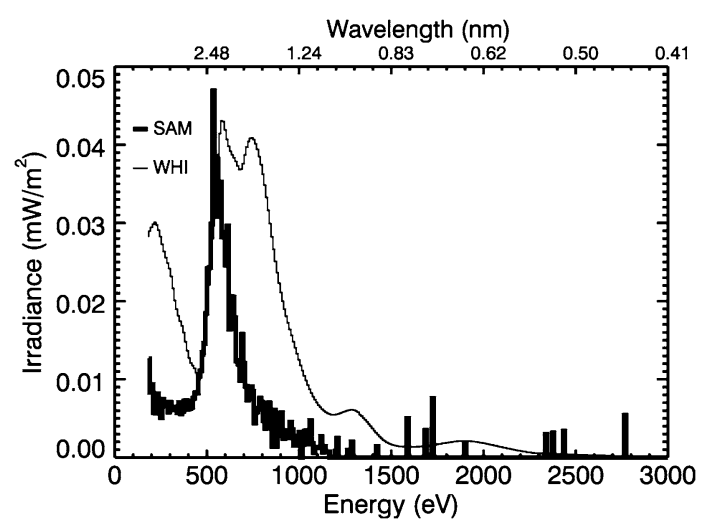

The solar spectrum generated by SAM is much lower than the WHI reference spectrum. Over the range 177 to $1240 \mathrm{eV}$ ( 1 to $7 \mathrm{~nm}$ ), the total SAM irradiance is $5.74 \times 10^{-5} \mathrm{~W} \mathrm{~m}^{-2}$, while that of WHI is $1.57 \times 10^{-4} \mathrm{~W} \mathrm{~m}^{-2}$. The ESP irradiance for the $0.1-7 \mathrm{~nm}$ band is $9.9 \times 10^{-5} \mathrm{~W} \mathrm{~m}^{-2}$, and the XPS broadband result for 0.1 to $7 \mathrm{~nm}$ is $9.3 \times 10^{-5} \mathrm{~W} \mathrm{~m}^{-2}$. These later values are more accurate as a broadband measurement than the WHI "model" spectrum. Nonetheless, the SAM irradiance is much lower than any of these other results; for example, SAM is $42 \%$ lower than the ESP irradiance. This large difference may be related to processing photon events for the SAM solar measurements, but not doing photon event processing for the SAM calibration data. Of particular concern, the solar data might have multiple photon events that are being interpreted as single photon events. Some indication for this concern is that the derived gain for solar measurements indicated $25 \%$ differences for the SAM gain value (see Section 7.1). Additional studies are planned to help resolve these differences in the SAM calibrations and solar measurement.

\section{Conclusions}

These EVE calibration results, along with the first rocket results with the prototype EVE, confirm that the EVE instruments are exceeding design requirements. As discussed in more detail by Woods et al. (2010), MEGS is designed to measure the solar EUV irradiance from 5 to $105 \mathrm{~nm}$ with $0.1 \mathrm{~nm}$ spectral resolution, ten-second temporal cadence, and better than $25 \%$ accuracy. The accuracy of the MEGS responsivity ranges from $1 \%$ to $10 \%$, with the larger uncertainties occurring at wavelengths where there are low signals during SURF calibrations or associated with larger corrections for grating higher-order contributions. Application of the SURF calibrations to the solar measurement yields irradiance accuracy of $5 \%$ to $20 \%$, again wavelength dependent and with larger uncertainties at wavelengths where there are low signals.

These calibrations also highlight some limitations of the EVE instruments. There are a couple of spectral regions where MEGS has low responsivity; consequently, those regions have less accurate calibration and irradiance results. These regions include the 5 to $6 \mathrm{~nm}$ range for MEGS A1, the 15 to $17 \mathrm{~nm}$ range for MEGS A1 and A2, and 35 to $42 \mathrm{~nm}$ range for MEGS A2 and B. From these multiple SURF calibration sets, we have learned how to better select the optimal SURF conditions, such as its beam energy, for each MEGS instrument, thus leading to more accurate responsivity results for the more recent calibrations of the rocket-prototype EVE instruments. These lessons learned will be transferred to the 
flight EVE with the first EVE underflight rocket calibration, planned for three months after SDO launches. The MEGS P spectral bandpass is the least accurate part of its algorithm, estimated to have $10 \%$ uncertainty but perhaps larger. Overlap with SOLSTICE can provide in-flight calibration for MEGS $P$ to improve upon its pre-flight calibrations. The SAM solar-irradiance results have significant differences between its spectrum and the modeled spectrum used in the analysis of XPS broadband data. Some of this difference could be related to the model predictions, but part of this difference might be related to the photon event extraction algorithm. More extended measurements, than just one rocket measurement, are expected to help clarify the differences and lead to improvements for the SAM calibrations and algorithms.

The results from EVE, along with the solar-cycle minimum results from the rocket launch in April 2008, will significantly improve upon the understanding of the solar EUV variability over all time scales from solar-cycle variations over a period of years, to solar-rotation variations that happen on the order of days, as well as solar flares on time scales of seconds to hours. The anticipated EVE irradiance results coupled with the solar-imaging results from SDO's other experiments will advance our understanding of irradiance variations and eruptive events such as flares. These advances are then expected to improve upon Earth's ionospheric and thermospheric models for space-weather operations, to better track satellites, and to manage communication and navigation systems. Furthermore, the EVE observations will provide an important extension of the solar EUV irradiance record currently being made by the SOHO and TIMED satellites.

Acknowledgements The authors would like to express their appreciation to Mitch Furst and the staff at NIST SURF III for all of their help throughout this calibration process, and special thanks go to Mitch Furst for reviewing this paper. We would also like to thank the many dedicated LASP scientists and engineers who designed and built EVE, and the many who also spent many weeks at the NIST calibration facility to obtain these calibration results. We also express special thanks to Vanessa George at LASP for supporting the preparation of this manuscript. We thank the referee for their helpful comments and suggestions. The solar irradiance results from 14 April 2008 can be obtained from the LASP Interactive Solar Irradiance Datacenter (LISIRD, http://lasp.colorado.edu/lisird). The EIT image is from the SOHO/EIT Consortium; SOHO is a joint ESA and NASA program. This research is supported by NASA contract NAS5-02140.

Open Access This article is distributed under the terms of the Creative Commons Attribution Noncommercial License which permits any noncommercial use, distribution, and reproduction in any medium, provided the original author(s) and source are credited.

\section{References}

Arp, U., Friedman, R., Furst, M.L., Makar, S., Shaw, P.-S.: 2000, Metrologia 37, 357.

Bailey, S.M., Woods, T.N., Eparvier, F.G., Solomon, S.C.: 2006, Adv. Space Res. 37, 209.

Chamberlin, P.C., Woods, T.N., Eparvier, F.G.: 2002, Opt. Eng. 45, 063605.

Chamberlin, P.C., Hock, R.A., Crotser, D.A., Eparvier, F.G., Furst, M., Triplett, M.A., Woodraska, D., Woods, T.N.: 2007, SPIE Proc. 6689, 66890N.

Chamberlin, P.C., Woods, T.N., Crotser, D.A., Eparvier, F.G., Hock, R.A., Woodraska, D.L.: 2009, Geophys. Res. Lett. 36, L05102.

Crotser, D.A., Woods, T.N., Eparvier, F.G., Ucker, G., Kohnert, R., Berthiaume, G., Weitz, D.: 2004, SPIE Proc. 5563, 182.

Crotser, D.A., Woods, T.N., Eparvier, F.G., Triplett, M.A., Woodraska, D.L.: 2007, SPIE Proc. 6689, $66890 \mathrm{M}$.

Didkovsky, L., Judge, D., Wieman, S., Woods, T., Jones, A.: 2010, Solar Phys. doi:10.1007/s11207-0099485-8.

Henke, B.L., Gullikson, E.M., Davis, J.C.: 1993, At. Data Nucl. Data Tables 54, 181.

Hinteregger, H.E., Fukui, K., Gilson, B.R.: 1981, Geophys. Res. Lett. 8, 1147. 
Rottman, G.J., Woods, T.N., Sparn, T.P.: 1993, J. Geophys. Res. 98, 10667.

Saloman, E.B.: 1975, Appl. Opt. 14, 1391.

Triplett, M.A., Crotser, D.A., Woods, T.N., Eparvier, F.G., Chamberlin, P.C., Berthiaume, G.D., Weitz, D.M., Vest, R.E.: 2007, SPIE Proc. 6689, 668900.

Westhoff, R.C., Rose, M.K., Gregory, J.A., Berthiaume, G.D., Seely, J.F., Woods, T.N., Ucker, G.: 2007, SPIE Proc. 6686, 668604.

Woods, T.N., Rottman, G.: 2005, Solar Phys. 230, 375.

Woods, T.N., Rottman, G., Vest, R.: 2005, Solar Phys. 230, 345.

Woods, T.N., Tobiska, W.K., Rottman, G.J., Worden, J.R.: 2000, J. Geophys. Res. 105, 27195.

Woods, T.N., Eparvier, F.G., Bailey, S.M., Chamberlin, P.C., Lean, J., Rottman, G.J., Solomon, S.C., Tobiska, W.K., Woodraska, D.L.: 2005, J. Geophys. Res. 110, A01312.

Woods, T.N., Chamberlin, P.C., Harder, J.W., Hock, R.A., Snow, M., Eparvier, F.G., Fontenla, J., McClintock, W.E., Richard, E.C.: 2009, Geophys. Res. Lett. 36, L01101.

Woods, T.N., Chamberlin, P., Eparvier, F.G., Hock, R., Jones, A., Woodraska, D., Judge, D., Didkosky, L., Lean, J., Mariska, J., McMullin, D., Warren, H., Berthiaume, G., Bailey, S., Fuller-Rowell, T., Sojka, J., Tobiska, W.K., Viereck, R.: 2010, Solar Phys. doi:10.1007/s11207-009-9487-6. 